\title{
Les relations entre l'ANASE et l'Afrique: vers un partenariat renouvelé ?
}

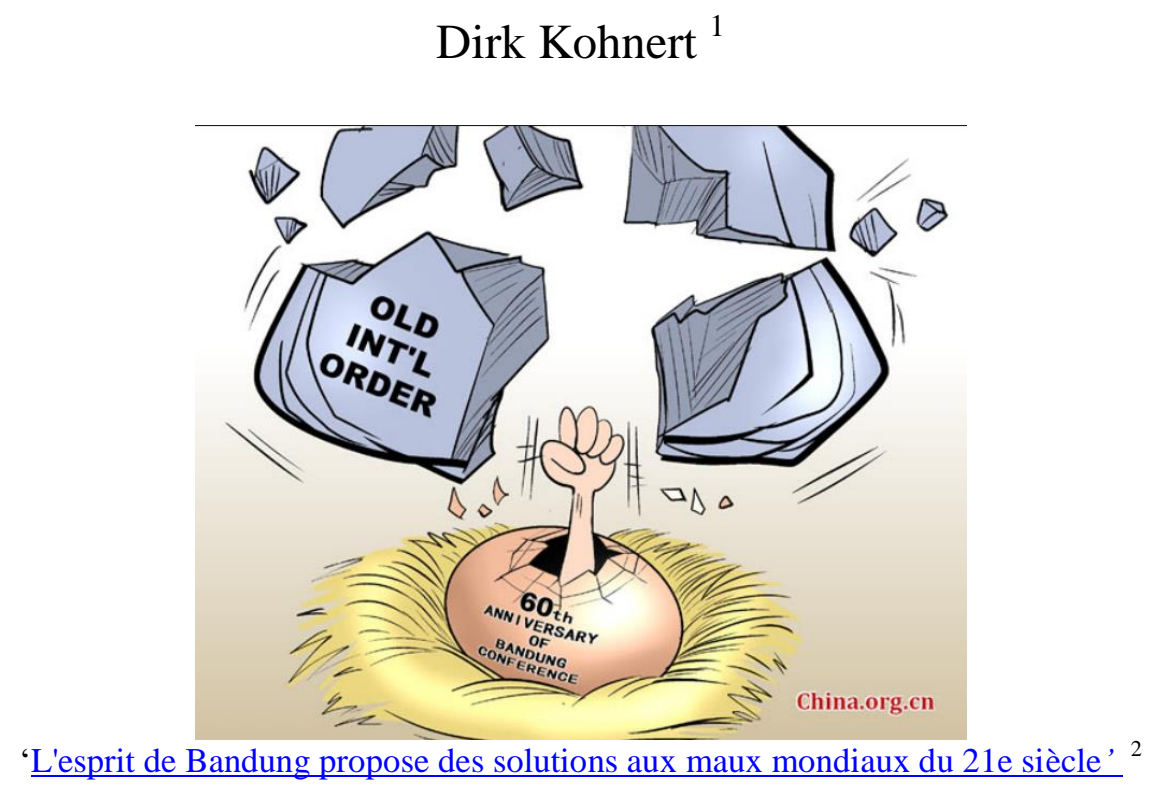

Résumé : Le sommet de l'ASEAN d'octobre 2021 a montré l'importance géopolitique accrue de la region indo-pacifique. Aujourd'hui, l'ANASE est l'organisation régionale la plus performante d'Asie et la deuxième au monde derrière l'UE. La création du Nouveau partenariat stratégique Asie-Afrique (NAASP) plus de 15 ans auparavant (2005) visait à raviver l'esprit de Bandung du mouvement des non-alignés de 1955. Cette fois en mettant davantage l'accent sur les liens économiques. En 2013, ces pays comptaient environ 620 millions d'habitants soit $8,8 \%$ de la population mondiale. Ils voulaient combattre le colonialisme et le néocolonialisme en promouvant la coopération économique et culturelle afro-asiatique. Presque tous les pays membres ont acquis leur souveraineté et leur indépendance politique dans les années 1960 et 1970, à l'exception de la Palestine. Cependant, les conséquences de la conférence de Bandung ont également favorisé des développements négatifs, notamment la polarisation des pays asiatiques, le renforcement de l'autoritarisme politique et les interventions régionales. En outre, la plupart des pays ont continué à faire face à des défis économiques et politiques, notamment la pauvreté, le fardeau de la dette, le retard, l'ignorance, la maladie et la dégradation de l'environnement. Leur accès aux marchés des pays industrialisés restait également limité. Au niveau mondial, le NAASP a reçu peu d'attention jusqu'à présent. Malgré la rhétorique de longue date de la solidarité Asie-Afrique, l'Asie et l'Afrique manquent encore de liens institutionnels et commerciaux formels. Bien que le commerce interrégional ait augmenté, l'Afrique est restée une petite partie de l'ASEAN avec seulement environ 2\% de son marché total. Les principaux pays commerçants de l'ASEAN avec l'Afrique, étaient la Thaillande, l'Indonésie et Singapour, tandis que l'Afrique du Sud, le Nigéria et l'Égypte étaient les plus grands marchés d'importation africains.

Mots clés: ANASE [ASEAN], NAASP, Asie du Sud-Est, Afrique, croissance économique, commerce international, zone de libre échange, union douanière, mouvement des non-alignés, Indonésie, Afrique du Sud, Thaïlande, Singapour, Nigeria, Egypte

JEL-code: F13, F15, F36, F54, N15, N17, O17, O19, O53, O55, Z13

\footnotetext{
1 Dirk Kohnert, directeur adjoint (retraité), Institut des Affaires Africaines, GIGA, Hamburg. Projet: 15 Novembre 2021.

${ }^{2}$ L'esprit de Bandung propose des solutions aux maux mondiaux du 21e siècle, par Mammo Muchie et Yan Hui. Shanghai Daily (Quotidien de Shanghai), 27 avril 2015 - Source: Cartoon Popular \& China Internet Information Center, China.org.en, 2015.
} 


\section{Introduction}

l'Association des nations de l'Asie du Sud-Est (ASEAN ou ANASE en Français) constitue l'une des zones de libre-échange (ZLE) les plus vastes et les plus importantes au monde. C'est l'organisation régionale la plus performante d'Asie et la deuxième dans le monde derrière l'UE. Ses dirigeants ont créé, en étroite coopération avec des partenaires internationaux, certains des plus grands forums et blocs multilatéraux du monde, notamment la Coopération économique pour Asie-Pacifique, le Sommet de l'Asie orientale et le Partenariat régional économique global. Au total, l'ASEAN est composée de dix États membres, Brunei, Cambodge, Indonésie, Laos, Malaisie, Myanmar (Birmanie), Philippines, Singapour, Thaïlande et Viêtnam (ASEAN Free Trade Area, Wikipedia). Ces pays comptaient environ 620 millions d'habitants, soit 8,8\% de la population mondiale (en 2013).

L'attention mondiale pour le sommet (virtuel) de trois jours de l'ASEAN du 26 au 28 octobre 2021, présidé par le Sultanat de Brunei, a montré l'importance géopolitique accrue du bassin indo-pacifique. Pourtant, l'ASEAN a refusé d'être perçue uniquement à cause de la concurrence stratégique entre les États-Unis et la Chine. Beaucoup craignaient de devenir l'herbe piétinée sous les pieds des éléphants combattants dans une confrontation croissante ou même une nouvelle guerre froide (Fähnders, 2021). Cependant, tous les États d'Asie du SudEst n'étaient pas également alarmés par la montée en puissance de la Chine. Après tout, la Chine était également la plus grande partenaire commercial de l'ASEAN. Entre autres, l'ASEAN a participé à la nouvelle initiative chinoise «la Ceinture et la Route » (nouvelle route de la soie) et à des accords commerciaux, comme le Partenariat régional économique global ( $\underline{\text { RCEP }}$ ), signés par 15 pays d'Asie-Pacifique en novembre 2020, y compris les dix membres de l'ASEAN, comme la Chine, la Corée du Sud, le Japon, la Nouvelle-Zélande et l'Australie. Il s'agirait du plus grand accord de libre-échange au monde (Xinhua, 2021).

Carte 1 : les 10 états membres de l'ASEAN, 2021

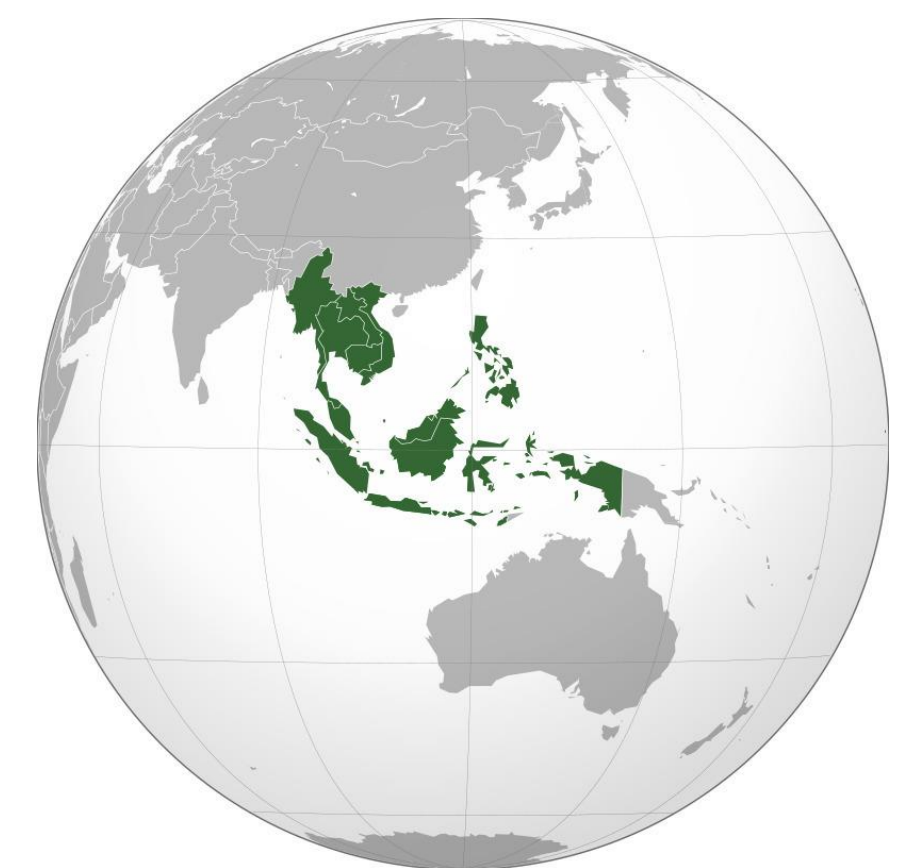

Source: ASEAN (orthographic projection), Wikimedia.commons

\footnotetext{
${ }^{3}$ Les ratifications d'au moins six états membres de l'ANASE et trois pays non-membres de l'ANASE étaient nécessaires pour atteindre le seuil d'entrée en vigueur du RCEP. Les pays qui ont jusqu'à présent terminé les procédures de ratification sont la Chine, Brunei, la Thaïlande, Singapour, le Japon et le Cambodge.
} 
Les différences de systèmes politiques, entre régimes démocratiques et autocratiques, ont apparemment joué un rôle moindre pour les Asiatiques du Sud-Est que, par exemple, pour les Européens.

Cependant, le sommet d'octobre a été éclipsé par une décision sans précédent. Le chef militaire du Myanmar, Min Aung Hlaing, qui avait renversé le gouvernement civil le ler février 2021, a été empêché de participer au sommet. Il s'agit de la sanction la plus sévère que l'ASEAN ait jamais infligée à un État membre. La raison de cette mesure inhabituelle de l'ASEAN, jusqu'alors connue pour sa politique de non-ingérence, était l'échec du régime militaire du Myanmar à mettre en œuvre le plan de paix convenu avec l'ASEAN en avril 2021 pour mettre fin à la crise sanglante qui avait déclenché le coup d'État (Aljazeera, 2021). Le coup d'État avait mis fin à l'expérience de démocratie de courte durée du Myanmar. La lauréate du prix Nobel Aung San Suu Kyi, qui avait été Premier ministre (Conseillère d'État du Myanmar) et ministre des Affaires étrangères de 2016 à 2021, fait désormais face à un tribunal de la junte avec des peines potentiellement lourdes. Le Myanmar avait rejoint l'association en 1997 sous un précédent gouvernement militaire.

Outre la crise du Myanmar, les questions à l'ordre du jour du sommet comprenaient les demandes controversées de la Chine concernant la mer de Chine méridionale où Pékin avait transformé de petites îles et atolls en bases militaires au cours de la dernière décennie. Pourtant, la Chine, le Viêtnam et d'autres pays d'Asie du Sud-Est avaient des revendications qui se chevauchaient. Enfin, la gestion de la pandémie de COVID-19 a été discutée. Les réunions au Brunei se déroulaient en ligne en raison des restrictions de voyage liées à Corona. De manière significative, le président américain Joe Biden, qui souhaitait rallier des soutiens dans la région contre une Chine montante, a également participé à un sommet États-UnisASEAN le 26 octobre ainsi qu'à un sommet auquel ont participé le Premier ministre chinois Li Keqiang et d'autres dirigeants mondiaux le 27 octobre 2021 (France-24, 2021).

Les racines historiques de l'ASEAN ont été jetées après la Seconde Guerre mondiale par la Conférence de Bandung, la première conférence afro-asiatique à grande échelle, organisée par l'Indonésie, le Myanmar (Birmanie), Ceylan (Sri Lanka), l'Inde et le Pakistan du 18 au 24 avril, 1955, à Bandung, Java occidental, Indonésie. Les 29 nations participant à la Conférence de Bandung étaient préoccupées par les implications de la guerre froide. Avec leur opposition prononcée au colonialisme et leur plaidoyer en faveur du neutralisme politique, ils ont créé le Mouvement des non-alignés. À l'occasion du 50e anniversaire de la conférence de Bandung en 2005, les dirigeants asiatiques et africains, éclairés par «l'esprit de Bandung », se sont à nouveau réunis à Bandung et à Jakarta, lançant le Nouveau partenariat stratégique afroasiatique (NAASP) pour promouvoir une nouvelle politique stratégique, coopération économique et culturelle entre les deux continents (Alatas, 2005; Albert, 2021). Au niveau mondial, le NAASP a reçu peu d'attention jusqu'à présent, contrairement aux relations commerciales de l'Asie avec d'autres continents, qui se sont cristallisées dans des institutions telles que la Réunion Asie-Europe (ASEM) et le Forum de coopération Asie de l'EstAmérique latine (FEALAC) (GIS-Asie, 2016).

Serait-ce que le manque d'attention internationale pour le NAASP était principalement dû au fait que la nouvelle coopération stratégique afro-asiatique s'est avérée être une lettre morte, un tigre de papier, en raison d'un manque de détermination politique de part et d'autre ? Y aura-til des chances réalistes d'améliorer les relations ASEAN-Afrique dans un avenir prévisible?

Dans ce qui suit, les réponses à ces questions seront discutées. Cependant, l'analyse des performances économiques des États membre de l'ASEAN en Afrique (et l'envers) avait été 
entravée jusqu'à présent par la diversité politique, économique, sociale et culturelle prononcée de l'ASEAN et de l'Afrique. Le PIB par habitant de Singapour, par exemple, était environ 50 fois plus élevé que celui du Cambodge et du Myanmar, et 30 fois celui du Laos. L'Indonésie représentait près de $40 \%$ de la production économique de l'ASEAN, tandis que le Myanmar était encore en train de se doter d'institutions. Par conséquent, toute tentative d'analyser les interactions des acteurs politiques de manière unidimensionnelle serait trompeuse (Vaidyanathan, 2016). L'étude de la performance à travers des études de cas, comme dans l'approche suivante, pourrait être une alternative valable pour illustrer les grandes tendances du développement du partenariat ASEAN-Afrique et des relations commerciales.

Après une esquisse de l'histoire des relations ASEAN-Afrique, en mettant l'accent sur les relations commerciales et les investissements directs à l'étranger (IDE), un bref historique de la performance du NAASP sera présenté. Par la suite, des études de cas approfondies de certains majeurs pays de l'ASEAN et d'Afrique ainsi que l'impact de la migration africaine vers l'ASEAN permettent d'étudier les perspectives de coopération ASEAN-Afrique.

\section{Histoire succincte des relations Asean - Afrique}

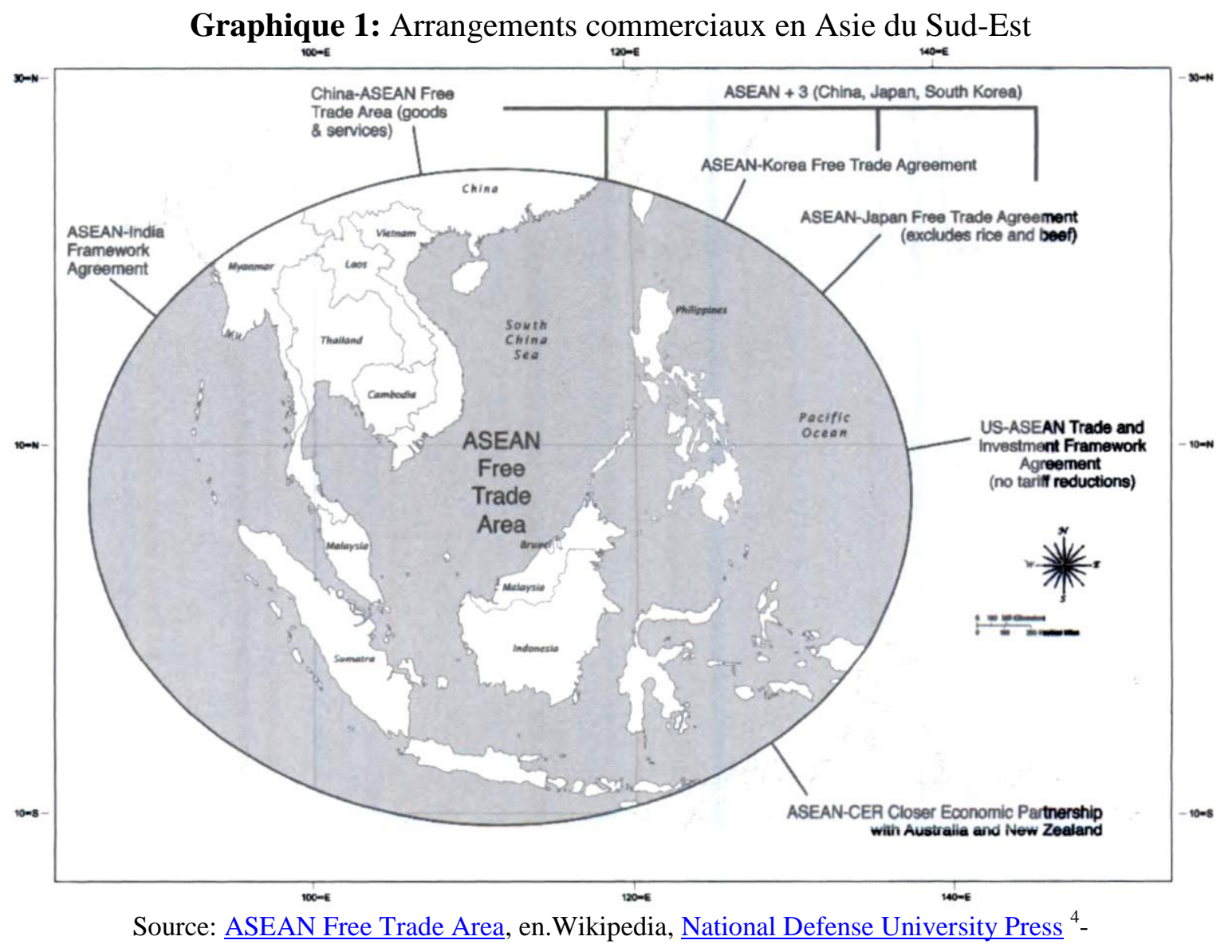

L'ASEAN constitue l'une des zones de libre-échange (ZLE) les plus vastes et les plus importantes au monde. C'est l'organisation régionale la plus performante d'Asie et la

\footnotetext{
${ }^{4}$ Source: Vaughn, B., McDonald, S. M., Clad, J., National Defense University. Center for Strategic Research. (2011). The borderlands of Southeast Asia: geopolitics, terrorism, and globalization. Washington, D.C.: Publié pour le Center for Strategic Research, Institute for National Strategic Studies par National Defense University Press.
} 
deuxième dans le monde derrière l'UE. Ses dirigeants ont créé, en étroite coopération avec des partenaires internationaux, certains des plus grands forums et blocs multilatéraux du monde, notamment la Coopération économique pour l'Asie-Pacifique, le Sommet de l'Asie orientale et le Partenariat régional économique global. Au total, l'ASEAN est composée de dix États membres, Brunei, Cambodge, Indonésie, Laos, Malaisie, Myanmar, Philippines, Singapour, Thaïlande et Viêtnam (ASEAN Free Trade Area, Wikipedia). En 2013, ces pays comptaient environ 620 millions d'habitants, soit $8,8 \%$ de la population mondiale.

Cependant, les conséquences de la conférence de Bandung ont également favorisé des développements négatifs, notamment la polarisation des pays asiatiques, le renforcement de l'autoritarisme politique et les interventions régionales. En outre, de nombreux pays ont continué à faire face à des défis économiques et politiques, notamment la pauvreté, le fardeau de la dette, l'arriération, l'ignorance, la maladie et la dégradation de l'environnement. Aussi, leur accès aux marchés des pays industrialisés est resté limité (GIS-Asie, 2016). Les divisions politiques entre les États membres de l'ASEAN reposaient en grande partie sur l'histoire fondatrice spécifique de l'association, en particulier les différences entre les premiers membres et les nouveaux venus. Les premiers membres étaient des pays à revenu intermédiaire (PRI) et au moins en partie démocratiques (Thaïlande, Philippines, Indonésie), tandis que les derniers étaient plutôt autoritaires (Birmanie et Viêtnam) et plus pauvres. Ces derniers s'opposaient à la politique de non-ingérence des premiers et préféraient se concentrer sur l'aide étrangère, et moins sur le commerce. Ces discordes ont affaibli l'alliance dans les négociations internationales et ont donné l'impression que l'ASEAN avait perdue son dynamisme et son agence (Association des nations de l'Asie du Sud-Est (ANASE), wikipédia).

\subsection{Le commerce ASEAN-Afrique et le développement de l'IDE}

Le commerce ASEAN-Afrique est passé de seulement 2,8 milliards US\$ en 1990 à 42,5 milliards US\$ en 2012, augmentant ainsi en moyenne de $14 \%$ par an. Cela a fait de l'Afrique le deuxième continent à la croissance la plus rapide pour le commerce de l'ASEAN autre que l'Asie (GIS-Asie, 2016).

Même si les pays de l'ASEAN étaient encore des principaux destinataires de l'IED, ils sont devenus une nouvelle source d'investissement pour de nombreuses économies en développement, en particulier dans la région de l'ASEAN. Les sorties globales d'IDE de l'ASEAN ont augmenté rapidement, passant de 8,97 milliards US\$ en 2000 à 56,36 milliards en 2013 (Sermcheep, 2017). Concernant les IDE, l'ASEAN était le troisième investisseur en Afrique, selon le gouvernement thaïlandais. Cependant, cela ne semble pas coller aux rapports sur l'investissement dans le monde de la CNUCED (UNCTAD) qui ont révélé que les États-

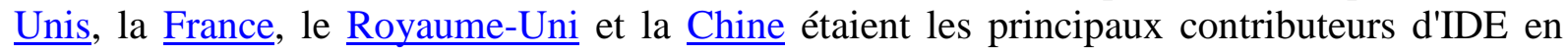
Afrique (par pays). En termes de capital total investi, y compris les investissements dans les projets d'infrastructure, la Chine est restée le plus important contributeur d'IDE en Afrique. Au cours de la période 2014-2018, la Chine a investi des fonds de plus de 72 milliards de US\$ en Afrique, créant plus de 137,000 emplois (AFR-IX, 2021). En dehors de cela, les structures d'investissement étaient principalement dus à des liens historiques. Par exemple, la France est restée un investisseur clé dans les régions de l'Afrique francophone où elle avait encore un avantage comparatif en raison de relations politiques et économiques établies et d'une langue et d'une culture commune. En dehors de cela, en 2020, les IDE ont chuté de $16 \%$ pendant la pandémie de Corona (AFR-IX, 2021). 
Jakarta avait établit des ambassades au Maroc, en Égypte, au Sénégal, au Nigeria, au Kenya, en Afrique du Sud et au Mozambique, ainsi qu'un consulat à Madagascar (Tarrosy. 2018). Ainsi, l'Afrique était également devenue une destination pour les investissements de l'ASEAN. L'investissement étranger direct (IED) de Singapour, par exemple, a atteint 15,9 milliards US\$ en 2012, ce qui en fait le plus grand investisseur de l'ASEAN en Afrique, suivi de la Malaisie et de l'Indonésie (GIS-Asie, 2016).

Plus de 200 entreprises de l'ASEAN opéraient en Afrique, principalement dans des secteurs tels que l'agro-industrie, la fabrication, le pétrole et le développement urbain. Cependant, dans l'ensemble, le marché africain est resté petit, avec 1,7 \% du total de l'ASEAN en 2012.

Tableau 1 : Commerce de marchandises de l'ASEAN avec 10 pays africains

\begin{tabular}{|c|c|r|r|r|}
\hline No. & Pays & \multicolumn{1}{c|}{ total } & \multicolumn{1}{c|}{ Exportation } & \multicolumn{1}{c|}{ Importation } \\
\hline 1 & $\begin{array}{c}\text { Afrique du Sud } \\
(\mathrm{ZA})\end{array}$ & 7.338 .065 .274 & 4.424 .809 .492 & 2.913 .255 .782 \\
\hline 2 & Nigeria (NG) & 4.683 .244 .760 & 1.592 .137 .328 & 3.091 .107 .432 \\
\hline 3 & Egypte (EG) & 3.499 .758 .664 & 3.000 .856 .725 & 492.901 .939 \\
\hline 4 & Libéria (LR) & 2.930 .721 .629 & 2.872 .239 .149$. & 58.482 .480 \\
\hline 5 & Angola (AO) & 2.338 .073 .583 & 558.596 .150 & 1.779 .477 .433 \\
\hline 6 & Côte d'Ivoire (CI) & 2.221 .365 .534 & 593301.371 & 1.628 .064 .163 \\
\hline 7 & Algérie (DZ) & 1.733 .920 .297 & 487.549 .380 & 1.246 .370 .917 \\
\hline 8 & Ghana (GH) & 1.698 .348 .242 & 973.145 .057 & 725.203 .185 \\
\hline 9 & Kenya (KE) & 1.448 .655 .917 & 1.286 .770 .155 & 161.885 .762 \\
\hline 10 & Congo (CG) & 875.640 .994 & 149.630 .498 & 726.010 .496 \\
\hline \multicolumn{7}{|c|}{ Exportations et importations de l'ASEAN en 2020, tous produits, en US\$ } \\
\hline \multicolumn{3}{|c|}{ Source: ASEAN Stats Data Portal } \\
\hline
\end{tabular}

Les principales nations commerçantes de l'ASEAN avec l'Afrique étaient la Thaïlande $(11,6$ milliards US\$), l'Indonésie (10,7 milliards US\$) et Singapour (9,5 milliards US\$) (GIF-Asie, 2016). Du côté africain, l'Afrique du Sud, le Nigéria et l'Égypte avaient le plus grand commerce global de la marchandise avec l'ANASE (voir le tableau 1). Sept États de l'ASEAN ont créé le comité ASEAN-Pretoria pour stimuler le commerce avec l'Afrique du Sud. Par ailleurs, le Viet Nam, le Cambodge et le Laos ont renforcé leurs liens économiques avec les pays africains francophones dans le cadre de l'Organisation Internationale de la Francophonie (OIF) (GIF-Asie, 2016).

Bien que la Chine soit devenue le partenaire commercial dominant de la plupart des pays africains, les pays membre de l'ASEAN, comme la Thaïlande, l'Indonésie, Singapour, la Malaisie et le Viêtnam figuraient également parmi les 10 premières destinations asiatiques des exportations africaines au cours des deux dernières décennies (graphique 3). Ainsi, chaque pays africain avait un partenaire commercial principal distinct en Asie. Ainsi, les exportations africaines vers l'Asie étaient positivement corrélées avec les exportations vers le reste du monde. Cela suggérait que contrairement au détournement des échanges, le commerce africain avec l'Asie complétait les exportations vers d'autres pays (Tang \& Zeng \& Zeufac, 2020). 
Graphique 2 : Commerce de marchandises de l'ASEAN avec 10 pays africains

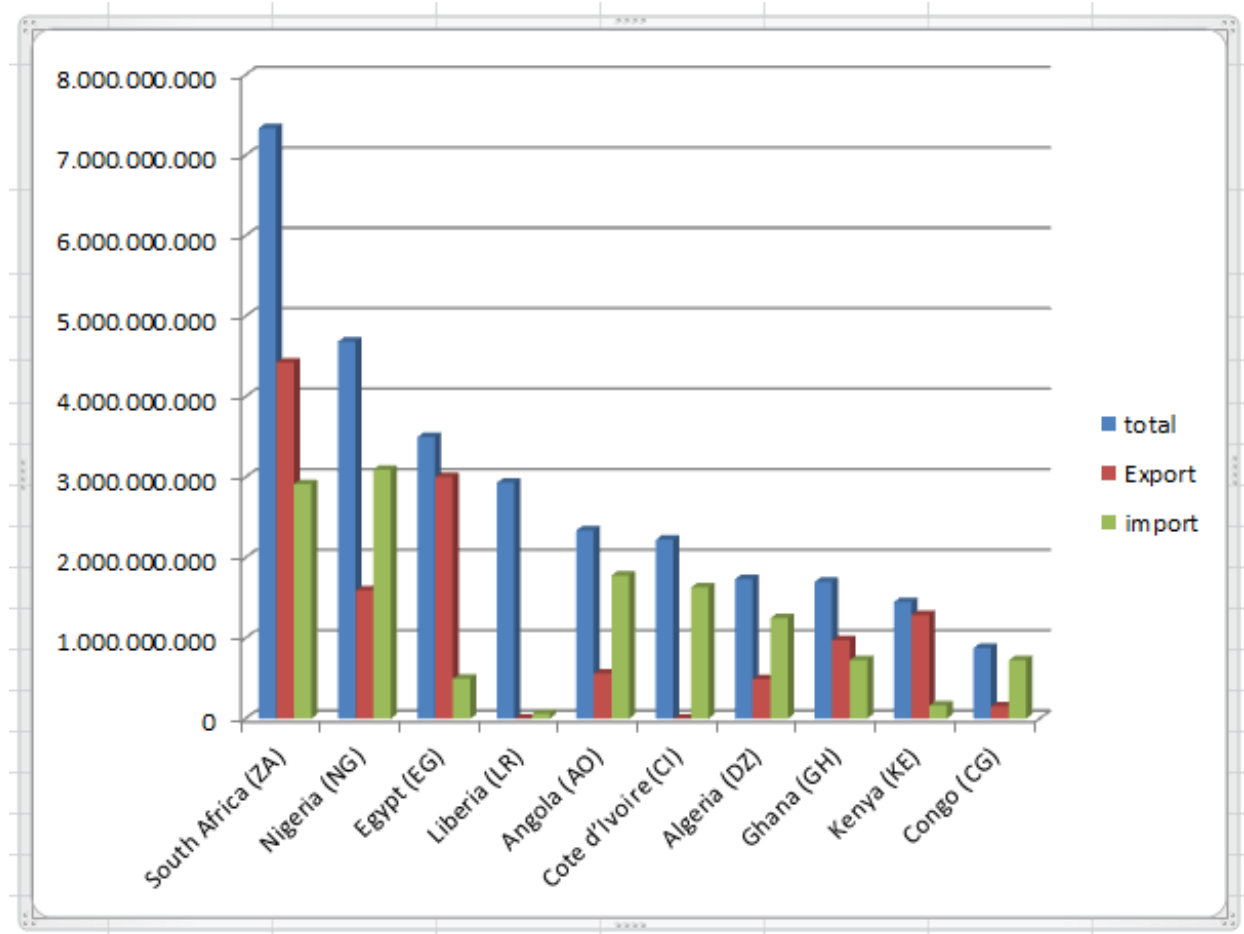

Les exportations et importations de l'ASEAN en 2020, tous produits, en US\$ Source: ASEAN Stats Data Portal

Selon l'opinion générale, l'intégration régionale en Asie du Sud-Est a été motivée par les marchés et les initiatives économiques privées, tandis que l'intégration régionale en Afrique subsaharienne (ASS) a été promue plutôt par des dirigeants et des États africains avec des intentions politiques intéressées et peu de résultats. Cependant, cela pourrait être trompeur, du moins en ce qui concerne le rôle prétendument dominant du marché dans le processus de développement de l'ASEAN.

Graphique 2 : Les 11 pays africaines exportant les plus importantes vers les destinations asiatiques, 2005 et 2015

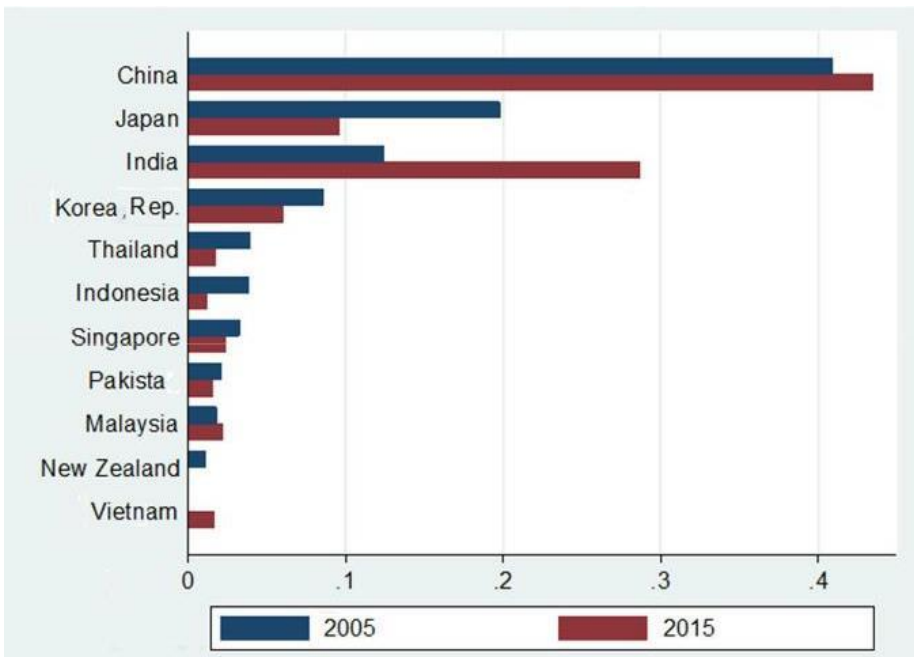

Source: Calculs IfW (Kiel), données de la base de données BACI World Trade du CEPII Tang, et al (2020) 
Très probablement, l'agence politique du Japon dans la promotion du commerce régional dans son hémisphère a été une base décisive pour le succès de l'ASEAN. Selon Coulibaly (Coulibaly, 2020), Tokyo a suivi le paradigme « vol d'oies sauvages » (Flying Geese), développé déjà avant la Seconde Guerre mondiale (1937), par Kaname Akamatsu, un universitaire japonais. Le paradigme s'est concentré sur la façon dont le développement pourrait être promu en Asie du Sud-Est par le Japon en tant que principal point d'ancrage régional. L'un des universitaires d'Akamatsu, l'économiste japonais Kiyoshi Kojima, a proposé dans les années 1980 que les accords régionaux selon le paradigme du "vol d'oies sauvages » pourraient aider à faciliter les transferts de technologie et la croissance de la productivité. Cela a jeté les bases théoriques de la Conférence du commerce et du développement du Pacifique et du Conseil de coopération économique du Pacifique (PECC) en 1980 et enfin de l'APEC de la Coopération économique pour l'Asie-Pacifique en 1989 (Coulibaly, 2020; Austine, 2019). Les liens entre les activités des sociétés multinationales japonaises en Asie de l'Est, par exemple, par la sous-traitance, l'octroi de licences, les coentreprises et l'investissement étranger direct (IDE), ont apparemment abouti à une situation gagnant-gagnant pour le Japon et les pays de l'ASEAN. L'IDE a commencé à croître dans les années 1970 et a considérablement augmenté au milieu des années 1980. Cela a facilité la restructuration économique du Japon tout en aidant simultanément la transformation structurelle de nombreux pays de l'ASEAN.

Deuxièmement, les pays africains pourraient tirer des leçons du rôle central des entreprises familiales dans le processus de développement de l'ASEAN (Coulibaly, 2020). Les grandes entreprises familiales diversifiées étaient, par exemple, des acteurs cruciaux de l'ASEAN Plus Trois (APT) qui comprend les États membres de l'ASEAN ainsi que la Chine, le Japon et la Corée du Sud. Elle a démarré en décembre 1997 et s'est institutionnalisée en 1999. Dans ces entreprises, la famille était souvent l'unité de gestion de base. Cela a permis des décisions rapides, de la loyauté, de la flexibilité et des frais généraux réduits. Enfin, il garantit la confiance, qui est une déterminante importante dans les transactions commerciales, notamment dans les contextes politiques à faible gouvernance (Coulibaly, 2020).

\subsection{Performance du nouveau partenariat stratégique Asie-Afrique (NAASP)}

L'ASEAN a abordé ses différences de développement ainsi que ses différents niveaux de préparation à l'ouverture des marchés, en mettant en œuvre une différenciation par le biais d'un mécanisme à plusieurs vitesses. Poussée par la Malaisie, membre le plus «arriéré », la formule a été baptisée dans le cadre de la libéralisation du secteur des services en 2002 le «principe 10 moins X ». Plus tard, elle a été nommée « formule ASEAN Minus X » et a ainsi été inscrite dans la Charte de l'ASEAN (Gaens, et al, 2020). Les exemples abondaient. Ainsi, des vitesses différentes figuraient en bonne place dans le «Protocole modifiant l'accord-cadre de l'ASEAN sur les services » en 2003, ainsi que dans la mise en œuvre du marché unique de l'aviation de l'ASEAN, autorisé en 2011. La différenciation était donc une caractéristique récurrente de l'intégration de l'ASEAN (Gaens, et al, 2020).

Le Nouveau partenariat stratégique Asie-Afrique (NAASP) a été fondé sur le Sommet AsieAfrique de 2005, auquel ont participé 106 pays, dont 54 pays asiatiques et 52 pays africains. Il visait à raviver l'esprit de la conférence de Bandung du mouvement des non-alignés de 1955. Cependant, contrairement aux années 1950, presque tous les pays membre avaient déjà acquis leur souveraineté et leur indépendance politique dans les années 1960 et 1970, à l'exception de la Palestine, toujours sous Occupation israélienne. Cette fois, les États membres ont mis 
davantage l'accent sur les liens économiques. Fondés sur la solidarité politique internationale, ils voulaient lutter contre le postcolonialisme en promouvant la coopération économique et socio-culturelle afro-asiatique. Lors d'une conférence de suivi à Jakarta du 12 au 13 octobre 2009, le NAASP a discuté d'une proposition de domaines prioritaires de coopération. Ces derniers, cependant, n'étaient pas nécessairement centrés sur les questions économiques, c'està-dire la lutte contre le terrorisme, la lutte contre le crime organisé transnational, la sécurité alimentaire et énergétique, les petites et moyennes entreprises ( $\underline{\mathrm{PME}})$, le tourisme, le réseau d'universités de développement afro-asiatiques, l'égalité des sexes, et l'autonomisation des femmes (NAASP, wikipedia).

Pourtant, depuis lors, le NAASP a fait peu de progrès. Bien que la déclaration du NAASP ait affirmé que les initiatives de développement afro-asiatiques déjà existantes pour l'Afrique, telles que la Conférence internationale de Tokyo sur le développement de l'Afrique (TICAD), le Forum sur la coopération sino-africaine (FOCAC) ou le Forum du sommet Inde-Afrique ( IASF) serait complété par le NAASP, il était difficile de rendre cet objectif opérationnel au profit de toutes les parties concernées (Tarrósy, 2016). Par exemple, il n'y avait pas de représentation institutionnalisée des partenaires africains dans les relations extérieures de l'ASEAN. Les critiques ont fait valoir que les deux parties, en particulier l'ASEAN, devraient surmonter les premiers obstacles au développement de leurs relations avec les pays africains en garantissant véritablement la volonté et l'engagement politique, avant la mise en œuvre du «partenariat stratégique»du NAASP envisagé en 2005, et par la formation de la Communauté économique de l'ASEAN (AEC) établie en 2015 lors du 27e sommet de l'ASEAN à Kuala Lumpur, en Malaisie. L'accent mis sur la coopération continent-pays a continué d'entraver l'objectif de longue date de formaliser le NAASP. Jusque-là, le NAASP risquait de ne subsister que pro forma (Dlamini, 2019).

\section{3. Études de cas}

\subsection{Relations commerciales indonésiennes - africaines}

L'Afrique en tant que partenaire commercial était encore assez lointaine pour de nombreux entrepreneurs privés indonésiens. Néanmoins, le commerce de l'Indonésie avec les pays africains était couvert par une présence diplomatique croissante. Les premières relations diplomatiques avec le Nigeria, le principal partenaire commercial, étaient déjà établies en 1965, lorsque Jakarta a ouvert une ambassade à Lagos. Le Nigeria a également été le premier pays d'Afrique subsaharienne à établir une ambassade à Jakarta en 1976 (Tarrósy, 2016). En 2016, Jakarta comptait 16 ambassades en Afrique, à savoir 6 en Afrique du Nord, soit au Maroc, en Algérie, en Tunisie, en Libye, en Egypte, au Soudan, et 10 en Afrique subsaharienne, en Afrique du Sud, au Nigeria, au Kenya, au Sénégal, en Ethiopie, en Tanzanie, Mozambique, Zimbabwe, Namibie et Madagascar. En outre, il gère un consulat général au $\underline{\text { Cap }}$ un attaché commercial au Caire et deux centres indonésiens de promotion du commerce (ITPC), l'un à Lagos et l'autre à Johannesburg (Tarrósy, 2016).

Le Nigéria était de loin le partenaire commercial le plus important, car l'Indonésie dépendait des importations de pétrole brut nigérian, suivie de l'Afrique du Sud et de l'Égypte. Les autres exportations nigérianes vers l'Indonésie comprenaient des matières premières en coton et en cuir. Les exportations indonésiennes vers le Nigéria, quant à elles, se concentrent sur l'huile de palme, les produits en papier, les produits pharmaceutiques, les pièces détachées automobiles, les savons et les produits électroniques. En outre, plus de 15 entreprises 
indonésiennes opéraient au Nigeria. L'un d'eux était Indofood qui a établi une usine de

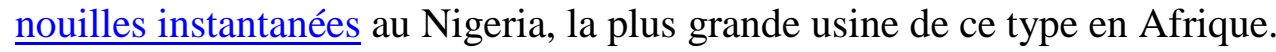

Graphique 3 : 10 principaux partenaires commerciaux africains de l'Indonésie en 2014 (en million US\$)

\begin{tabular}{|c|c|c|c|}
\hline Country & Exports & Imports & $\begin{array}{l}\text { Total } \\
\text { Trade } \\
\text { Value }\end{array}$ \\
\hline Nigeria & 648.61 & $3,306.30$ & $3,954.91$ \\
\hline $\begin{array}{l}\text { South Afri- } \\
\text { ca }\end{array}$ & $1,379.50$ & 498.49 & $1,877.99$ \\
\hline Egypt & $1,341.00$ & 145.93 & $1,486.93$ \\
\hline Algeria & 178.64 & 299.72 & 478.36 \\
\hline Angola & 189.66 & 209.28 & 398.94 \\
\hline Djibouti & 306.82 & 0.27 & 307.09 \\
\hline Benin & 213.11 & 31.10 & 244.21 \\
\hline Ghana & 212.27 & 28.72 & 240.99 \\
\hline Morocco & 82.19 & 140.26 & 222.45 \\
\hline Tanzania & 202.49 & 19.65 & 222.14 \\
\hline
\end{tabular}

Source: Tarrósy, 2016

Graphique 4 : Croissance des exportations indonésiennes vers l'Afrique, 1998 à 2016 (en million US\$)

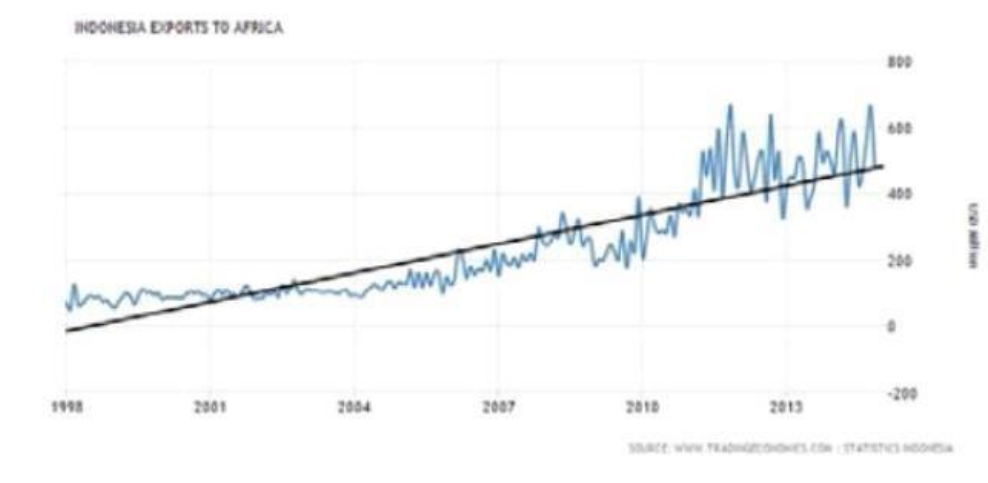

(Source: www.tradingeconomics.com / Statistics Indonesia)

Source: Tarrósy, 2016

Jakarta a encouragé la diversification du marché, notamment pour ses «produits nonpétroliers et gaziers », tels que l'huile de palme et les produits connexes, les textiles, les chaussures, les voitures et les composants automobiles, et l'électronique. Les 13 principaux marchés d'exportation d'Afrique subsaharienne à la croissance la plus rapide pour l'Indonésie en 2014 étaient la République Centrafricaine, la Somalie, la Mauritanie, Djibouti, le

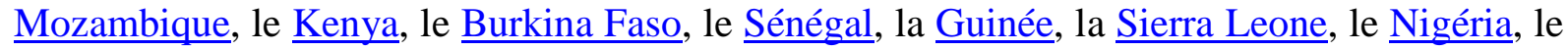
Congo (RDC) et l'Afrique du Sud (Tarrósy, 2016). 
Graphique 5 : Principales exportations indonésiennes par pays africain, 2009 et 2018
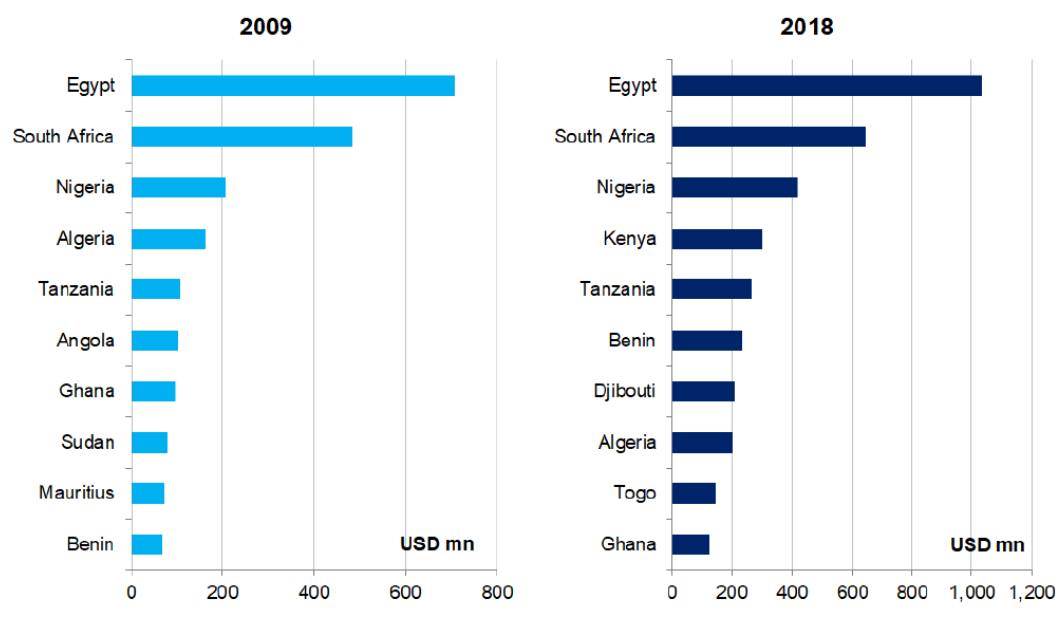

Source: Tanuwidjaja \&Handy, 2021

Graphique 6 : 10 principales exportations indonésiennes vers l'Afrique par la classification de produits, 2009 et 2018

2009

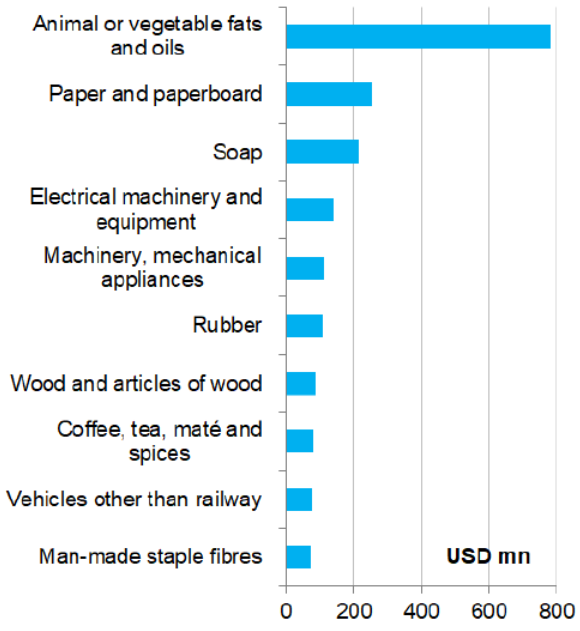

2018

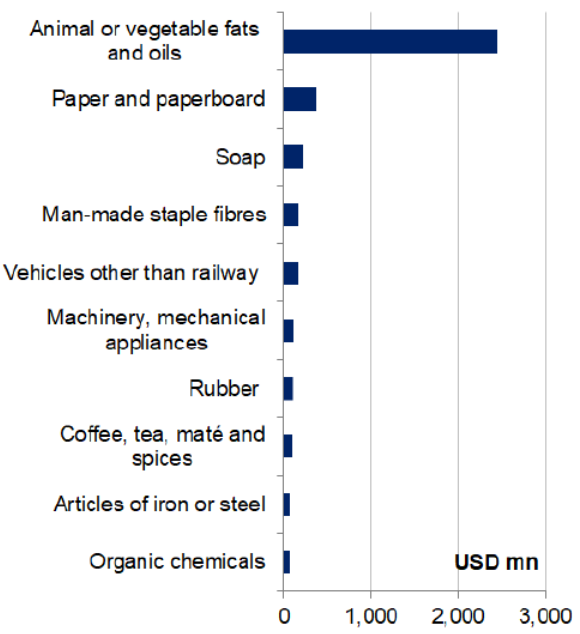

Source: International Trade Centre (ITC) database

\subsection{Relations commerciales Thaïlande - Afrique}

La part des importations thaïlandaises par continent n'était que de $2 \%$ pour l'Afrique en 2020, selon la base de données COMTRADE des Nations unies sur le commerce international. L'Angola, premier partenaire commercial africain d'importation de la Thaïlande, ne figurait qu'à la 25e place des pays importateurs. Les exportations de la Thaïlande par continent étaient légèrement plus importantes, avec $2,4 \%$ en 2020. L'Afrique du Sud était le partenaire commercial d'exportation le plus important, à la $21 \mathrm{e}$ position des partenaires commerciaux d'exportation les plus importants (Trading Economics, 2021). 
Graphique 7 : Exportations de la Thaïlande vers l'Afrique du Sud, 2010 - 2021

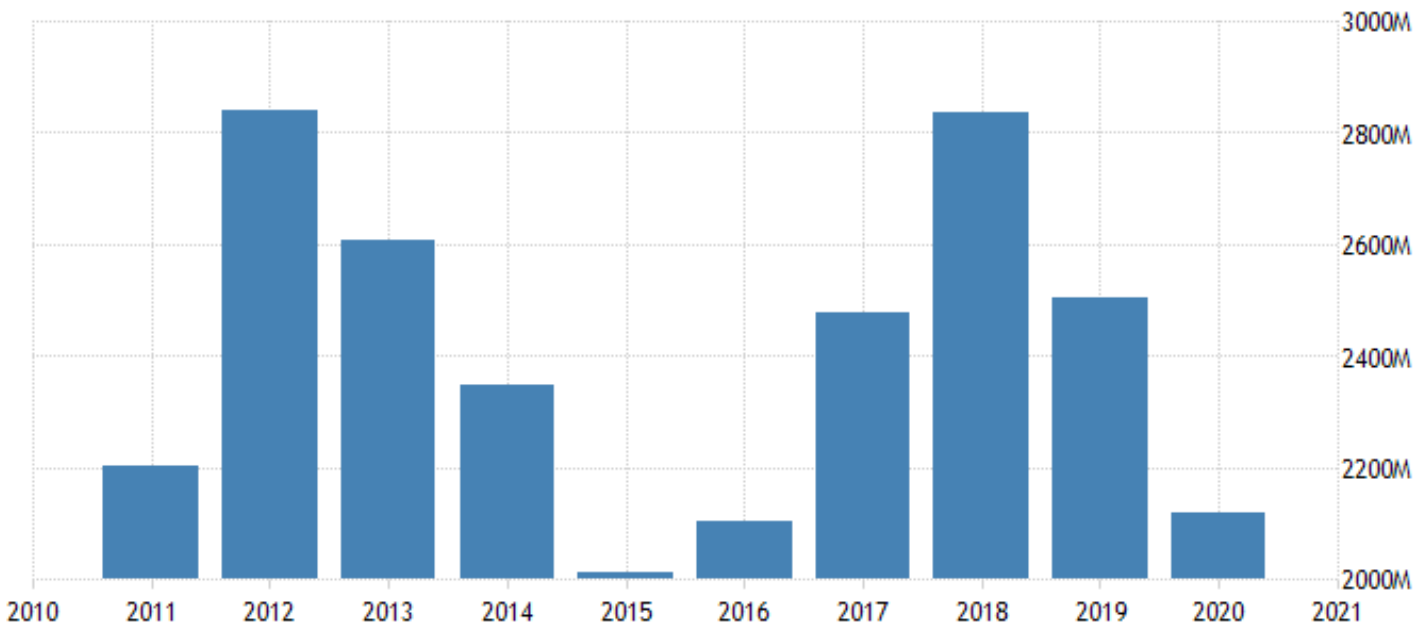

Source: Trading Economics, Thaïlande - Afrique du Sud

Graphique 8 : Exportations de la Thaïlande vers l'Afrique du Sud, catégories, 2020

\begin{tabular}{lll}
\hline Thailand Exports to South Africa & Value & Year \\
\hline Vehicles other than railway, tramway & $\$ 540.06 \mathrm{M}$ & 2020 \\
\hline Machinery, nuclear reactors, boilers & $\$ 494.33 \mathrm{M}$ & 2020 \\
\hline Cereals & $\$ 315.26 \mathrm{M}$ & 2020 \\
\hline Electrical, electronic equipment & $\$ 158.62 \mathrm{M}$ & 2020 \\
\hline Rubbers & $\$ 105.77 \mathrm{M}$ & 2020 \\
\hline Articles of iron or steel & $\$ 78.38 \mathrm{M}$ & 2020 \\
\hline Plastics & $\$ 63.53 \mathrm{M}$ & 2020 \\
\hline Meat, fish and seafood preparations & $\$ 57.62 \mathrm{M}$ & 2020 \\
\hline Organic chemicals & $\$ 41.99 \mathrm{M}$ & 2020 \\
\hline Optical, photo, technical, medical apparatus & Source: Trading Economics, Thaïlande - Afrique du Sud & 2020 \\
\hline
\end{tabular}

Depuis les années 2010, Jakarta a ouvert une nouvelle orientation diplomatique centrée sur l'Afrique. Ainsi, dans le cadre de la «Look West Policy» (Politique de regard vers l'ouest) en 2013, un partenariat Thaïlande-Afrique pour le développement durable a été lancé pour accroître l'engagement au-delà du voisinage asiatique immédiat du pays, avec l'Agence thaïlandaise de coopération internationale (TICA) agissant comme organisation clé au nom du gouvernement. En 2017, cette initiative avait été redéfinie pour signifier «donner la priorité à la coopération au développement basée sur la confiance, l'égalité et les intérêts mutuels avec les pays africains » (Tarrosy. 2018). À cette époque, la Thaïlande était la partenaire 
commercial le plus important de l'ASEAN avec l'Afrique et le plus grand fournisseur de riz du continent (Tarrosy. 2018).

Graphique 9 : Importations de la Thaïlande en provenance d'Angola

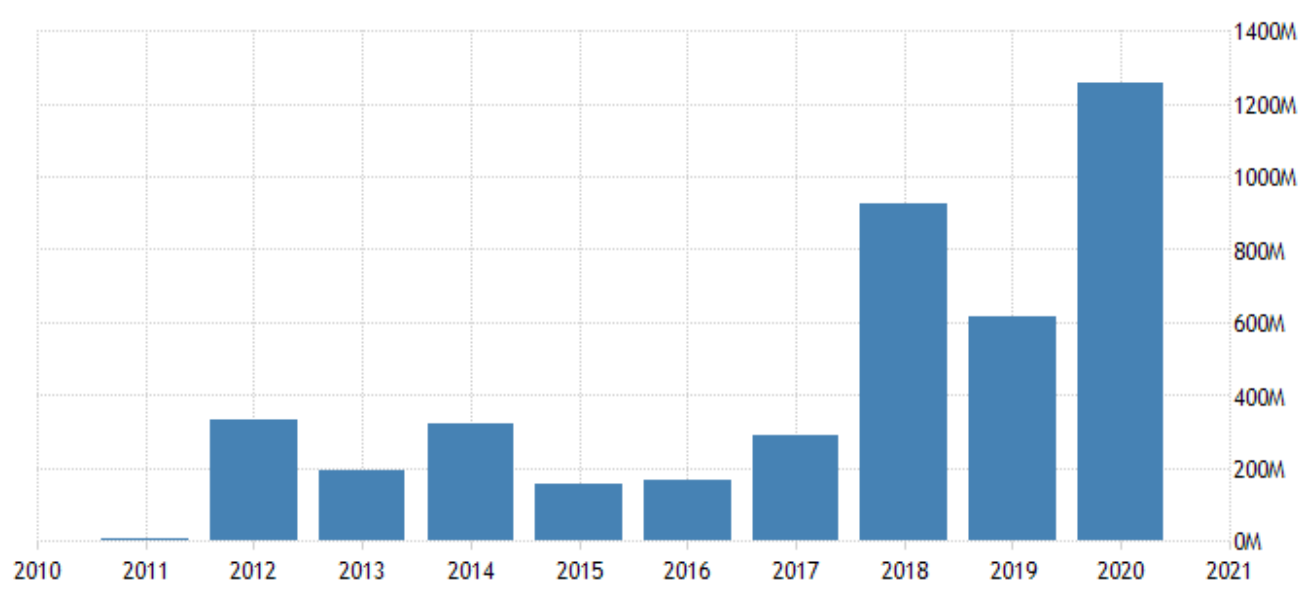

Source: $\underline{\text { Trading Economics }}$

L'ASEAN était le troisième investisseur (IDE) en Afrique, selon le gouvernement thaïlandais (cf. ci-dessus). Pourtant, il restait des obstacles substantiels à l'investissement thaïlandais en Afrique. L'incertitude sur les risques et les opportunités offertes par ce vaste marché était au premier plan. Par exemple, la seule société pharmaceutique thaïlandaise cotée en bourse traitant des marchés subsahariens était Mega, opérant à petite échelle, mais s'accélérant lentement au Kenya depuis la fin des années 1990. La société a progressivement étendu ses opérations au Ghana, au Kenya, au Nigeria, au Rwanda, en Tanzanie et en Ouganda. D'autres expansions étaient prévues en Éthiopie et au Soudan (Dhawan, 2017).

\subsection{Relations commerciales l'Afrique du Sud - ASEAN}

Les principaux partenaires commerciaux de l'Afrique du Sud en Asie étaient la Chine $(18,3 \%)$ et, dans une large mesure, la Thaïlande (cf. ci-dessus). Bien que les exportations de l'Afrique du Sud vers l'Asie aient été les plus élevées avec $34 \%$ parmi les continents d'exportation de l'Afrique du Sud, contre l'Europe (28\%), l'Afrique intérieure $(26 \%)$ et les Amériques $(11 \%)$, les pays de l'ASEAN occupent une place faible (Trading Economics). En 2020, le commerce total entre l'Afrique du Sud et l'ASEAN dépassait les 7 milliards de US\$ par an, l'ASEAN bénéficiant d'un excédent commercial. Les principaux partenaires commerciaux de l'Afrique du Sud au sein de l'ASEAN étaient Singapour, la Thaïlande et l'Indonésie. Les entreprises sud-africaines exportaient principalement des produits chimiques organiques et inorganiques, des minerais métalliques, des produits horticoles, du fer et de l'acier. Ils ont importé principalement des textiles et des vêtements, des chaussures, des produits électroniques, des huiles de pétrole raffinées, des produits automobiles et de l'huile de palm (Medina, 2020).

En général, les déclarations bien intentionnées ne manquaient pas, mais leur mise en œuvre était insuffisante. Le 19 novembre 2020, Pretoria a rejoint le traité d'amitié et de coopération (TAC) de l'ASEAN. Le TAC était un traité de paix signé déjà en 1976 entre les membres de l'ASEAN pour établir un ensemble de directives régissant les relations interétatiques dans la 
région. L'Afrique du Sud n'était que le troisième pays africain à avoir signé le traité après l'Egypte et le Maroc.

Graphique 10 : Exportations sud-africaines vs importations thaïlandaises, 2012 - 2020

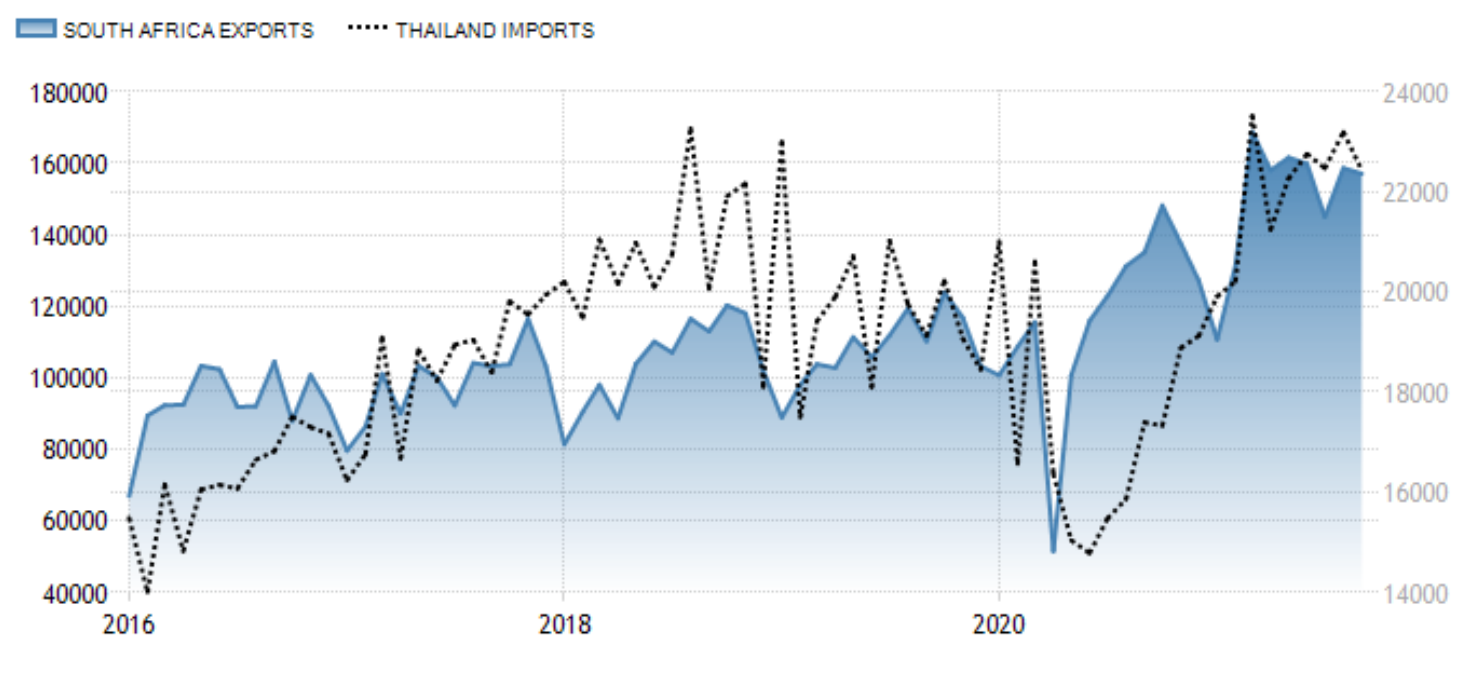

Source: (Trading Economics)

Graphique 11 : Importations sud-africaines vs exportations thaïlandaises, 2012 - 2020

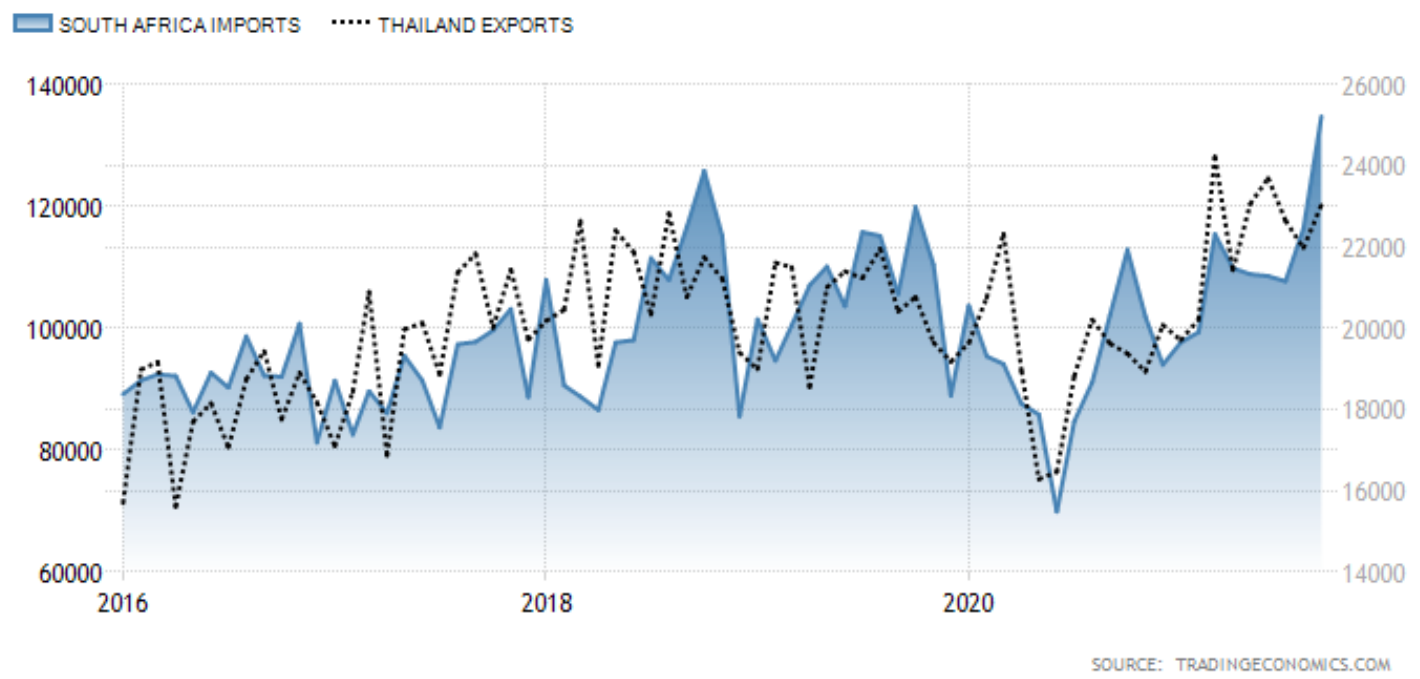

Source: (Trading Economics)

En 2012, Pretoria et Jakarta ont convenu de renforcer leurs relations bilatérales et de mener une étude conjointe pour explorer les opportunités commerciales en signant un comité commercial conjoint (JTC) à Jakarta. La même chose a été dite à propos d'autres États clés de l'ASEAN. Le Comité de Pretoria de l'ASEAN (APC), un comité de diplomates représentant les intérêts des États membres de l'ASEAN en Afrique du Sud, s'est réuni en 2014 pour discuter des moyens de renforcer la coopération multiforme entre l'ASEAN et l'Afrique du Sud (Dlamini, 2021). Pretoria, cependant, s'est efforcé de joindre les deux bouts, c'est-à-dire de trouver un moyen de tirer parti d'un accord de libre-échange (ALE), dans le cadre juridique 
actuel, au sein de l'Union douanière d'Afrique australe (SACU), du Marché commun de l'Afrique orientale et australe (SADC) et finalement au sein de la ZLECA (Fisher, 2020).

De plus, l'Afrique du Sud avait des opportunités d'exportation plus réalistes, par exemple pour commercer avec la Thaïlande, que l'inverse (à la fois en termes de nombre absolu d'opportunités disponibles et de leur «valeur inexploitée potentielle » (Rensburg, 2019). La Thaïlande figurait au 7e rang des importations sud-africaines par pays avec 2,14 milliards US\$ en 2020. Le Viêtnam était le 25e pays exportateur avec 602 millions US\$ en 2020. Quant aux importations sud-africaines, $47 \%$ provenaient du Viêtnam (Trading Economics).

\subsection{Relations commerciales Nigéria - ASEAN}

Les exportations des secteur de produits de base (pétrole et gaz naturel) étaient le principal moteur de la croissance et des recettes publiques du Nigéria depuis les années 1960. Il représentait plus de $91 \%$ des exportations totales. En 2014, $43 \%$ des ventes totales ont été réalisées en Europe, $29 \%$ en Asie, $13 \%$ en Amérique et $12 \%$ en Afrique (Trading Economics). Les relations commerciales bilatérales entre le Nigeria et le bloc de l'ASEAN ont atteint 7,7 milliards de US\$ en 2017 (Okeh, 2018). Les exportations nigérianes et sudafricaines, en général, ont évolué dans le même sens. Cependant, pendant la crise de Coronavirus, les exportations nigérianes ont pris du retard.

Graphique 12 : Exportations nigérianes et sud-africaines, 2012 - 2020

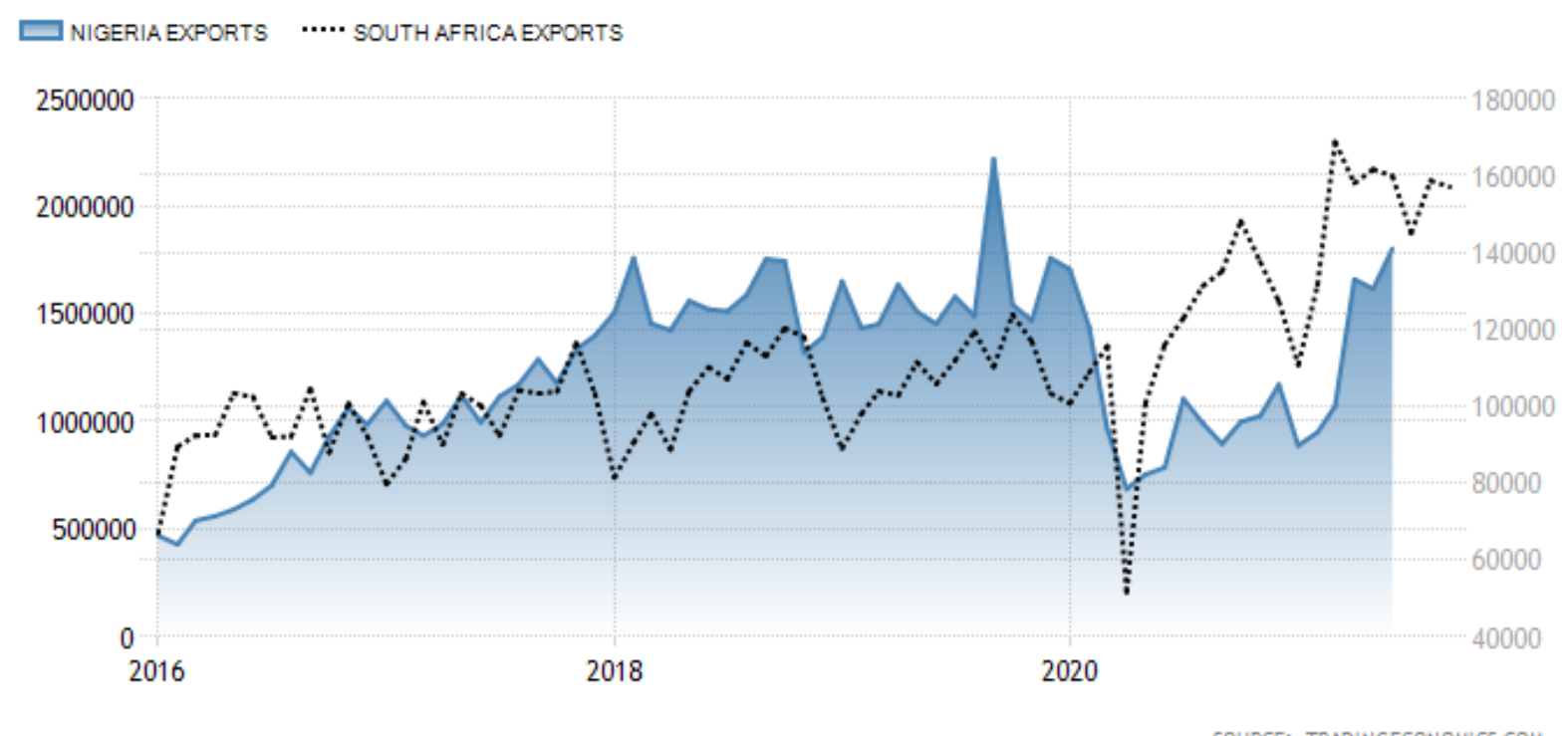

Source: (Trading Economics)

Les importations nigérianes comprenaient principalement des fournitures industrielles (27\% du total en 2014), des biens d'équipement (23\%), des aliments et boissons (17\%), des carburants et lubrifiants (14\%), des équipements et pièces de transport (12\%) et des produits de consommation (7 \%). La plupart des importations (43\%) provenaient d'Asie, principalement de la Chine, mais aussi des pays membre de l'ASEAN : $34 \%$ d'Europe, $15 \%$ d'Amérique et $7 \%$ d'Afrique. Bien que les importations du Nigeria aient été en général nettement inférieures à celles de l'Afrique du Sud, elles ont grimpé à peu près au même niveau pendant la crise de Coronavirus. 
Graphique 13 : Importations nigérianes et sud-africaines, 2012 - 2020

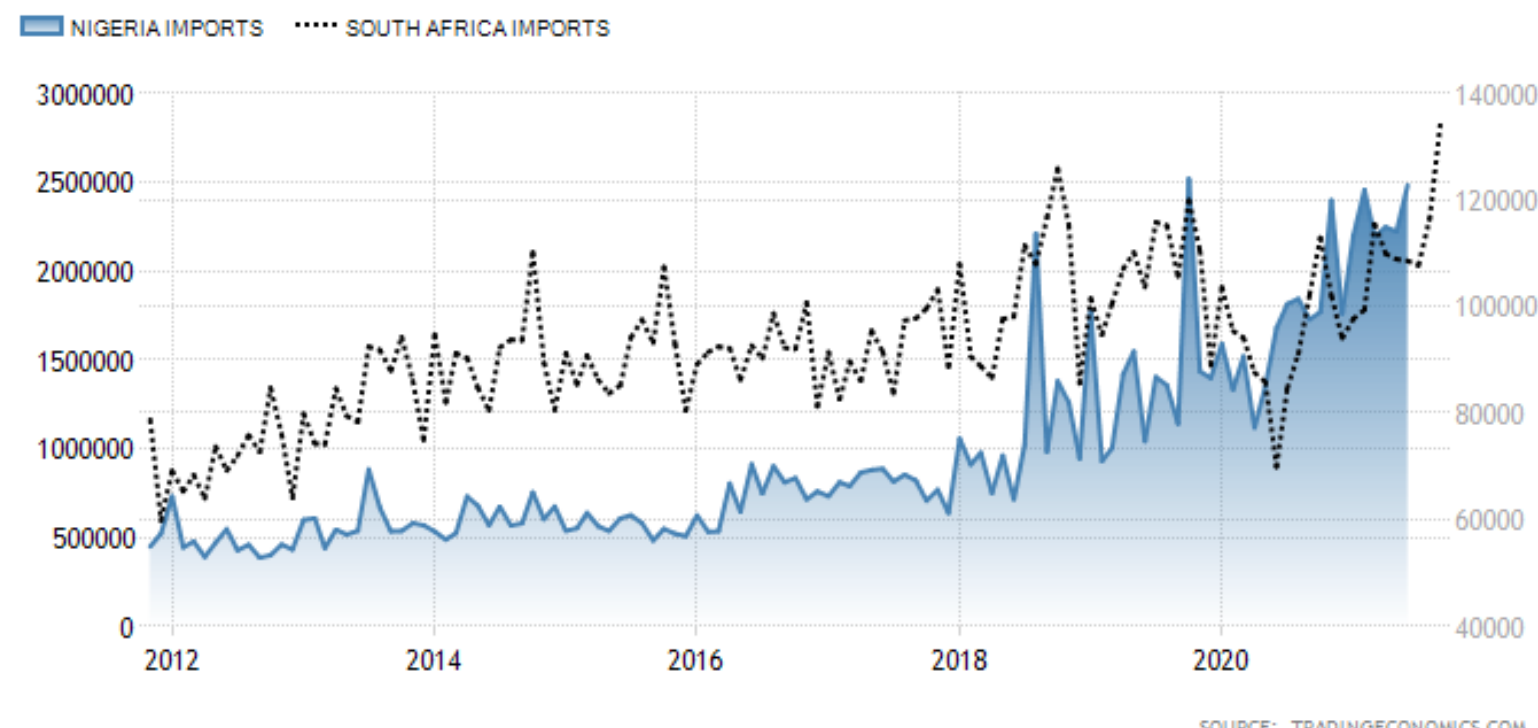

Source: (Trading Economics)

Cinq pays de l'ASEAN étaient représentés au Nigéria: la Malaisie, l'Indonésie, les Philippines, le Viêtnam et la Thaïlande (Okeh, 2018). Les deux membres de l'ASEAN, la Thaïlande et le Viêtnam, figuraient parmi les premières destinations asiatiques des exportations du Nigeria.

Graphique 14 : Principales destinations asiatiques des exportations du Nigeria, 2005 et 2015

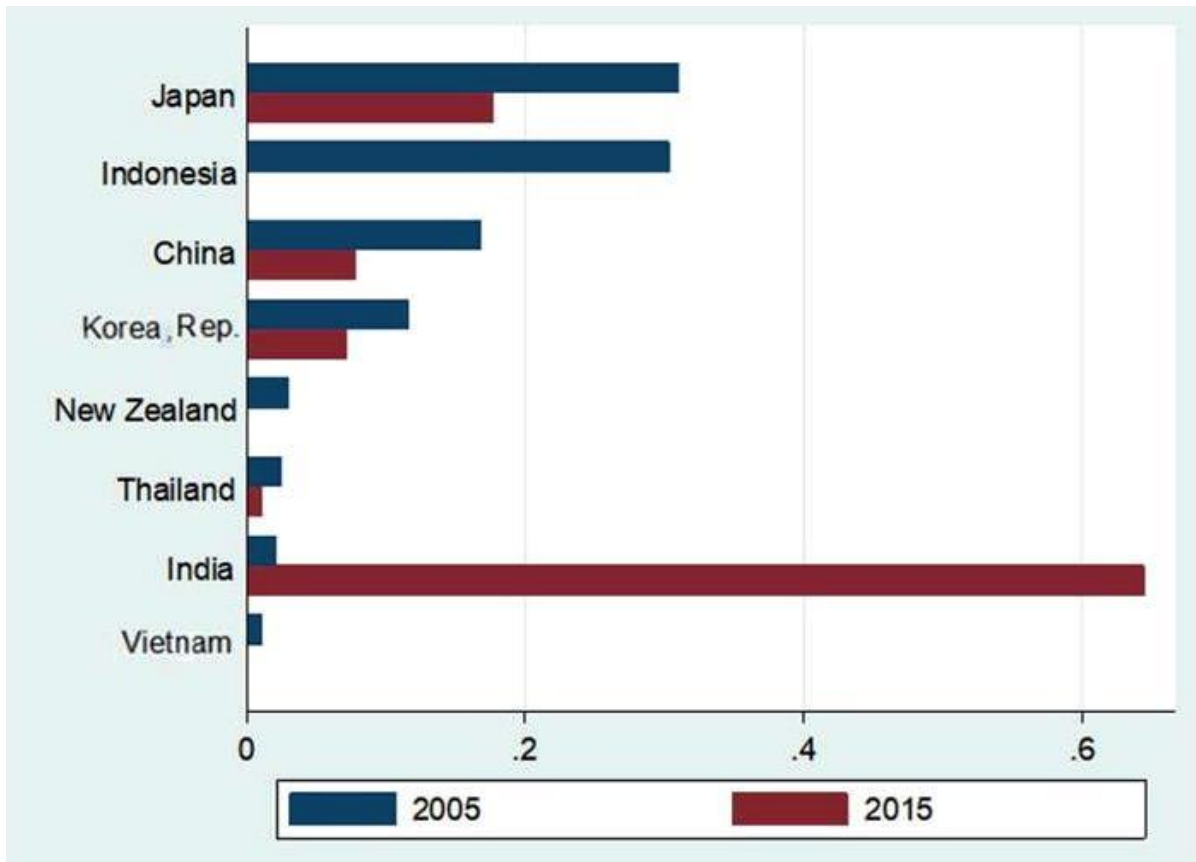

Source: Tang, et al (2020)

Le commerce bilatéral entre l'Indonésie et le Nigéria a triplé entre 2010 et 2014 (Tarrósy, 2016). Le Nigeria exporte vers les principaux pays importateurs de l'ASEAN, la Thaïlande et l'Indonésie, des pays développés dans le même sens et au même niveau. 
Graphique 15 : Commerce indonésien - nigérian, 2010 à 2014

(in million US\$)

\begin{tabular}{l|lll}
\hline Year & Export & Import & $\begin{array}{l}\text { Total Trade } \\
\text { Value }\end{array}$ \\
\hline 2010 & 316.86 & 921.59 & $1,238.45$ \\
2011 & 465.99 & $1,626.87$ & $2,092.86$ \\
2012 & 413.08 & $2,770.66$ & $3,183.74$ \\
2013 & 558.17 & $3,122.45$ & $3,680.62$ \\
2014 & 648.61 & $3,306.30$ & $3,954.91$ \\
\hline
\end{tabular}

(Source: Indonesia Central Bureau of Statistics (BPS),

Processed by Ministry of Trade)

Source: Tarrósy, 2016

Graphique 16 : Importations thaïlandaises et indonésiennes comparées, 2012-2020

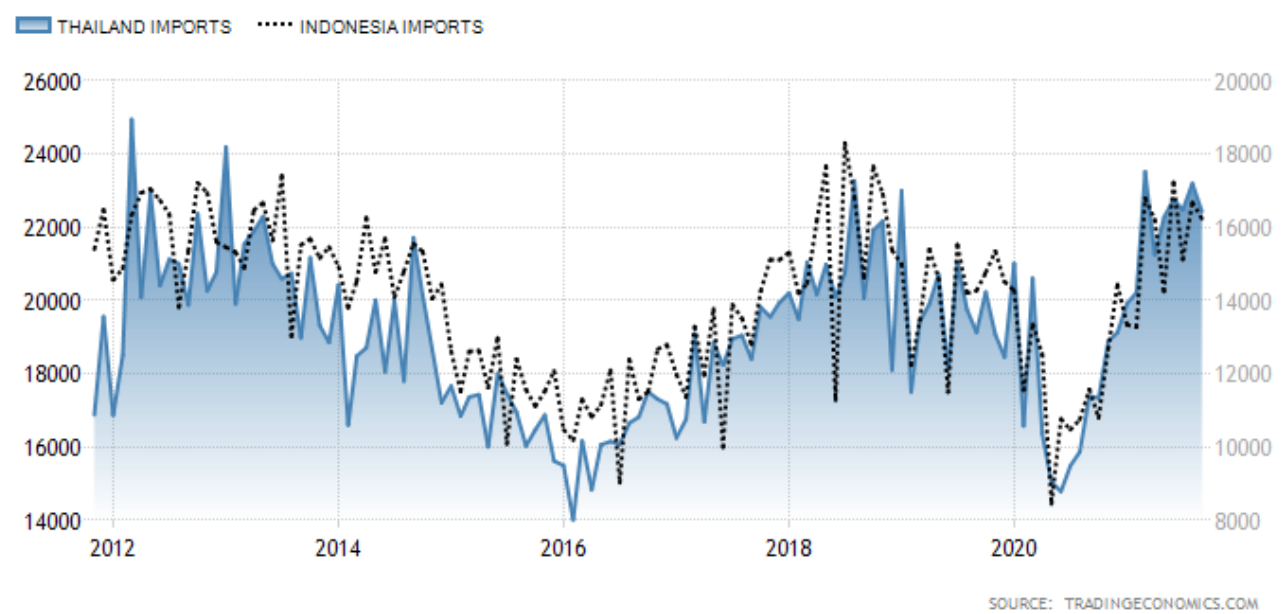

Source: (Trading Economics)

\subsection{Relations commerciales Egypte - ASEAN}

Située à côté des principales routes commerciales mondiales et à proximité des marchés arabes, africains, américains et européens, l'Égypte est une plaque tournante, reliant l'Afrique à l'Europe et à l'Asie, enfin et surtout grâce au canal de Suez. Sa position de hub régional est également soutenue par ses infrastructures relativement développées. Il n'est donc pas surprenant que les pays membre de l'ASEAN comme le Viêtnam, la Thaïlande et l'Indonésie, aient considérablement développé leurs relations commerciales extérieures ces derniers temps. De plus, il y avait des similitudes étroites entre le commerce extérieur du Nigeria et de l'Égypte avec les pays de l'ASEAN, par exemple, concernant le secteur des exportations, et, depuis la crise de Coronavirus, également concernant les importations.

Les médias égyptiens ont admiré les réalisations en matière de développement du Viêtnam et son rôle dans la région de l'ASEAN ainsi qu'un membre non-permanent du Conseil de sécurité des Nations Unies (CSNU) pour 2020-2021. L'Égypte était le deuxième partenaire commercial du Viêtnam en Afrique, son commerce bilatéral a atteint près de 500 millions de US\$ (VNA, 2020). Le commerce global entre l'Égypte et le Viêtnam s'élevait à 491 millions de US\$ en 2019. Les exportations vietnamiennes vers le marché égyptien étaient évaluées à 
458 millions de US\$, tandis que la valeur des importations vietnamiennes en provenance d'Égypte a atteint 33 millions de US\$ (Egypt Today staff, 2020).

En outre, la Thaïlande a discuté avec l'Égypte des moyens d'élargir leurs relations bilatérales en 2021. Les deux parties ont convenu de promouvoir la coopération dans des domaines clé, à savoir : (1) l'éducation, (2) le commerce et l'investissement, (3) le tourisme, (4) la culture et (5) la pêche, que l'Égypte souhaite tirer de la vaste expérience et de l'expertise de la Thaïlande (selon le Ministère thaïlandais des Affaires étrangères, 2021).

Il en est de même pour l'Indonésie. Les deux pays possédaient sans doute la plus grande puissance économique dans leurs régions respectives, sans parler d'Israël. L'Égypte était le 26e partenaire d'exportation de l'Indonésie en 2018. La valeur du commerce bilatéral a atteint 1,10 milliard de US\$, dont l'Indonésie a obtenu un excédent de 893,808 US\$.

Graphique 17 : Exportations nigérianes \& égyptiennes, 2012 - 2020

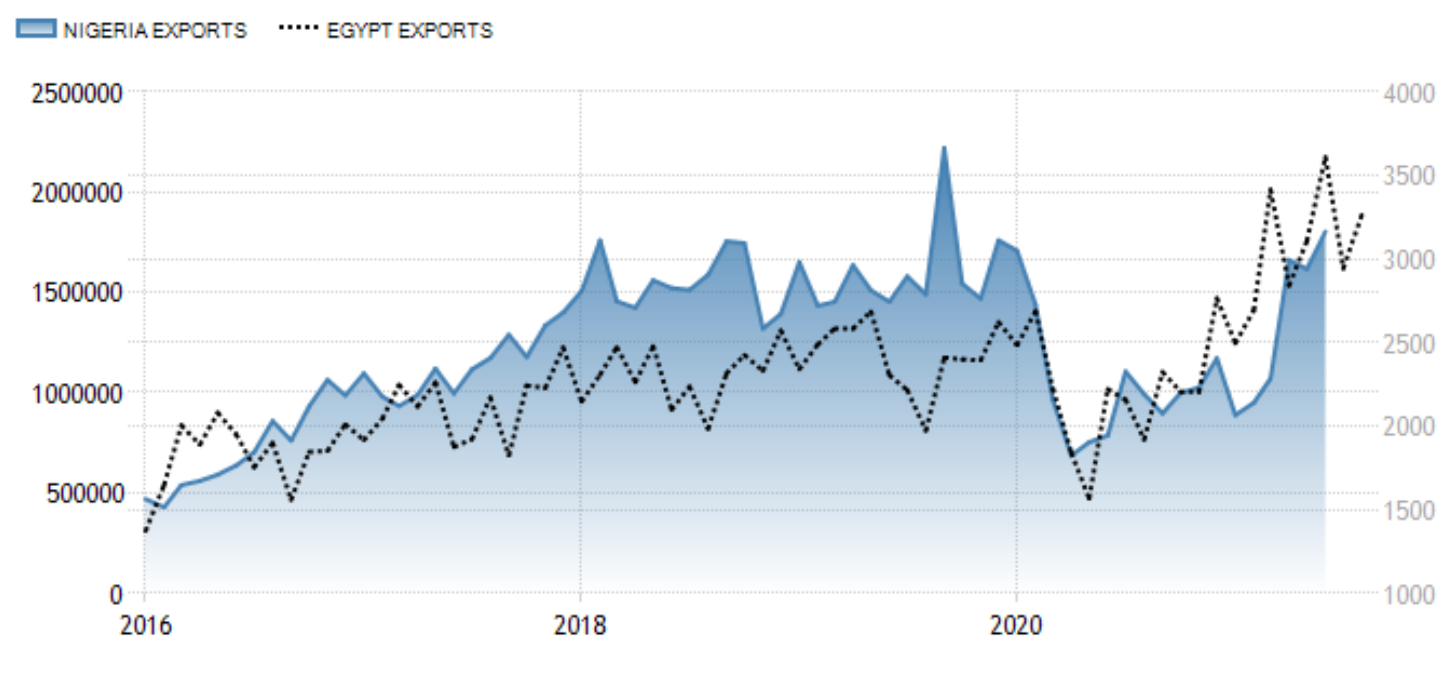

Source: (Trading Economics)

Graphique 18 : Importations nigérianes \& égyptiennes, 2012 - 2020

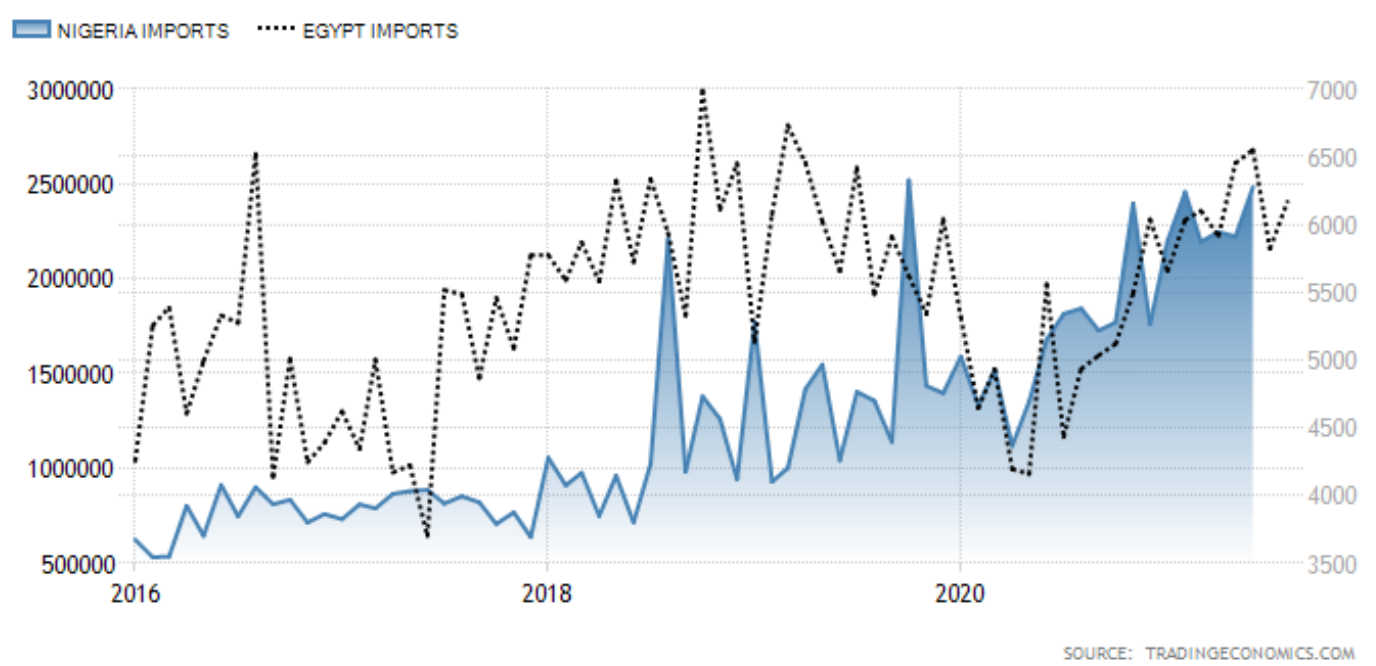

Source: (Trading Economics) 
Les échanges commerciaux entre l'Indonésie et l'Égypte en 2017 ont totalisé 1,253 milliards de US\$, contre 1,110 milliards de US\$ en 2016. Les exportations indonésiennes vers l'Égypte en 2017 étaient les plus élevées avec 1,505 milliards de US\$, soit une augmentation de près de $3 \%$ par rapport à 2016. Les principales exportations indonésiennes vers l'Égypte comprenaient de l'huile de palme, des pneus, du fil, du café et des pièces détachées automobiles. Les investissements indonésiens en Égypte en 2016 ont totalisé environ 50 millions de US\$. L'Égypte pourrait également se développer jusqu'au point d'entrée de l'huile de palme indonésienne sur le marché africain, car l'Égypte avait un accord de libre-échange (ALE) avec certains pays africains (SAWIT, 2018).

Près de 8,000 citoyens indonésiens résidaient en Égypte, dont 6,229 étaient des étudiants de l'enseignement supérieur, tandis que 463 travaillaient dans le secteur informel. Les deux gouvernements ont établi en 2019 une commission d'essai collective, un comité mixte sur le commerce, ainsi que d'autres partenariats dans les domaines de la pêche, de la santé, de l'énergie et de la défense. Afin d'améliorer l'accès au marché pour les produits des deux pays, l'Indonésie et l'Égypte souhaitaient lancer des études de faisabilité conjointes comme étape probatoire de leur accord commercial préférentiel (PTA) (Ministère des Affaires étrangères, Jakarta, 2019).

\section{Migration et relations ASEAN - Afrique}

Caricature 2 : «Les migrants par bateau en Asie »

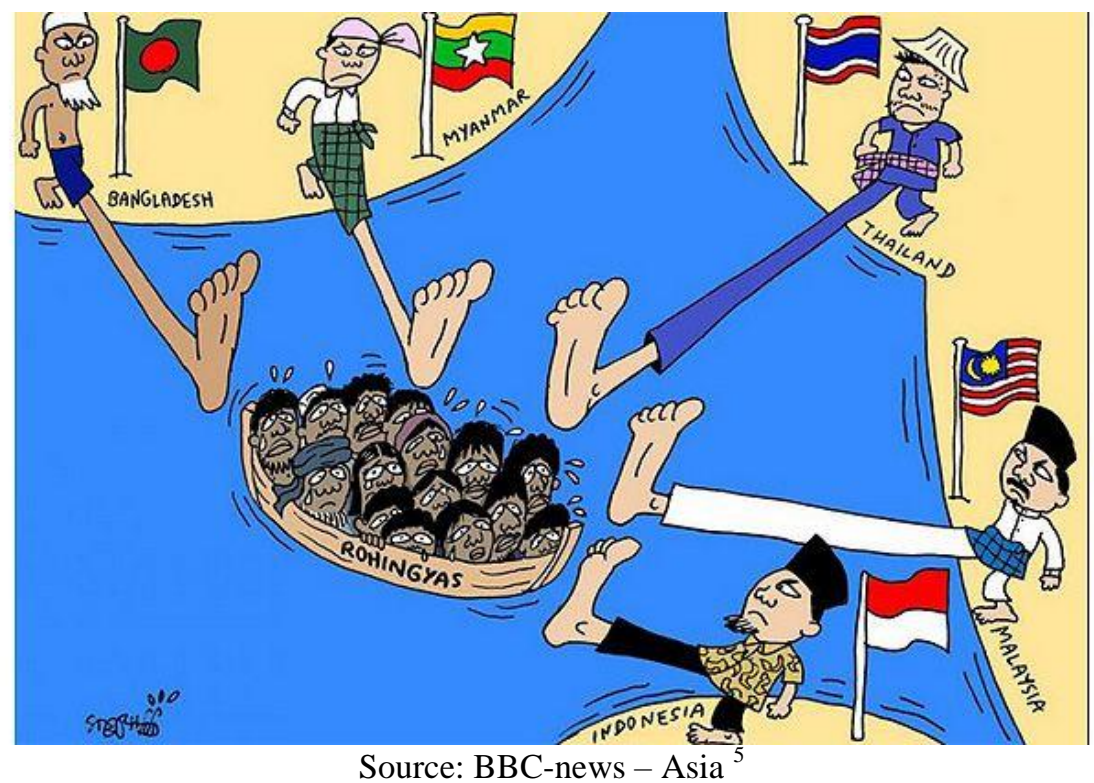

La migration de main-d'œuvre est devenue une caractéristique importante en Asie du Sud-Est au plus tard depuis les années 1970. Les politiques de l'État ont joué un rôle important dans la détermination des politiques migratoires spécifiques à chaque pays. Bien que la plupart des pays de l'ASEAN aient également envoyé des travailleurs migrants au Moyen-Orient et en Asie de l'Est, la migration intra-régionale était la plus importante. Concernant la dernière,

\footnotetext{
5 "Les migrants asiatiques en bateau : les médias de l'Asean s'inquiètent des surtraitements (Asia boat migrants: Asean media troubled overtreatment)", caricature montrant une cargaison de Rohingyas expulsés des côtes du Bangladesh, du Myanmar, de la Thaïlande, de l'Indonésie et de la Malaisie. N.A., BBC-news, 18 May 2015.
} 
deux schémas sont à distinguer (Yazid \& Septiyana 2019): Premièrement, les pays riverains du Mékong, c'est-à-dire le Cambodge, le Laos, le Myanmar et le Viêtnam, avec la Thaïlande comme principale destination des migrants. Deuxièmement, la région de la péninsule malaise de Brunei, de Malaisie et de Singapour avec des travailleurs migrants venant d'Indonésie et des Philippines. La Malaisie, par exemple, était un pays qui dépendait d'une main-d'œuvre bon marché pour l'agriculture et la fabrication. Par conséquent, la plupart des migrants venant en Malaisie étaient des travailleurs semi-qualifiés ou non qualifiés avec un faible niveau d'éducation. Singapour a été le premier pays de l'ASEAN à imposer une politique de maind'œuvre migrante pour le secteur manufacturier et des services personnels en raison de la menace d'une population vieillissante. De même, la majorité de ses travailleurs migrants n'étaient pas qualifiés. De nombreux travailleurs migrants dans la région ont été embauchés par des intermédiaires privés qui ont souvent demandé de l'argent pour leurs services aux migrants. En outre, les travailleurs migrants de l'ASEAN étaient principalement des femmes, car du côté de la demande, les profils d'emploi féminins, tels que les infirmières, les domestiques, les aides-soignants et les services hôteliers prévalaient (Yazid \& Septiyana 2019).

Graphique 19 : D'où viennent les migrants internationaux dans l'ASEAN ?

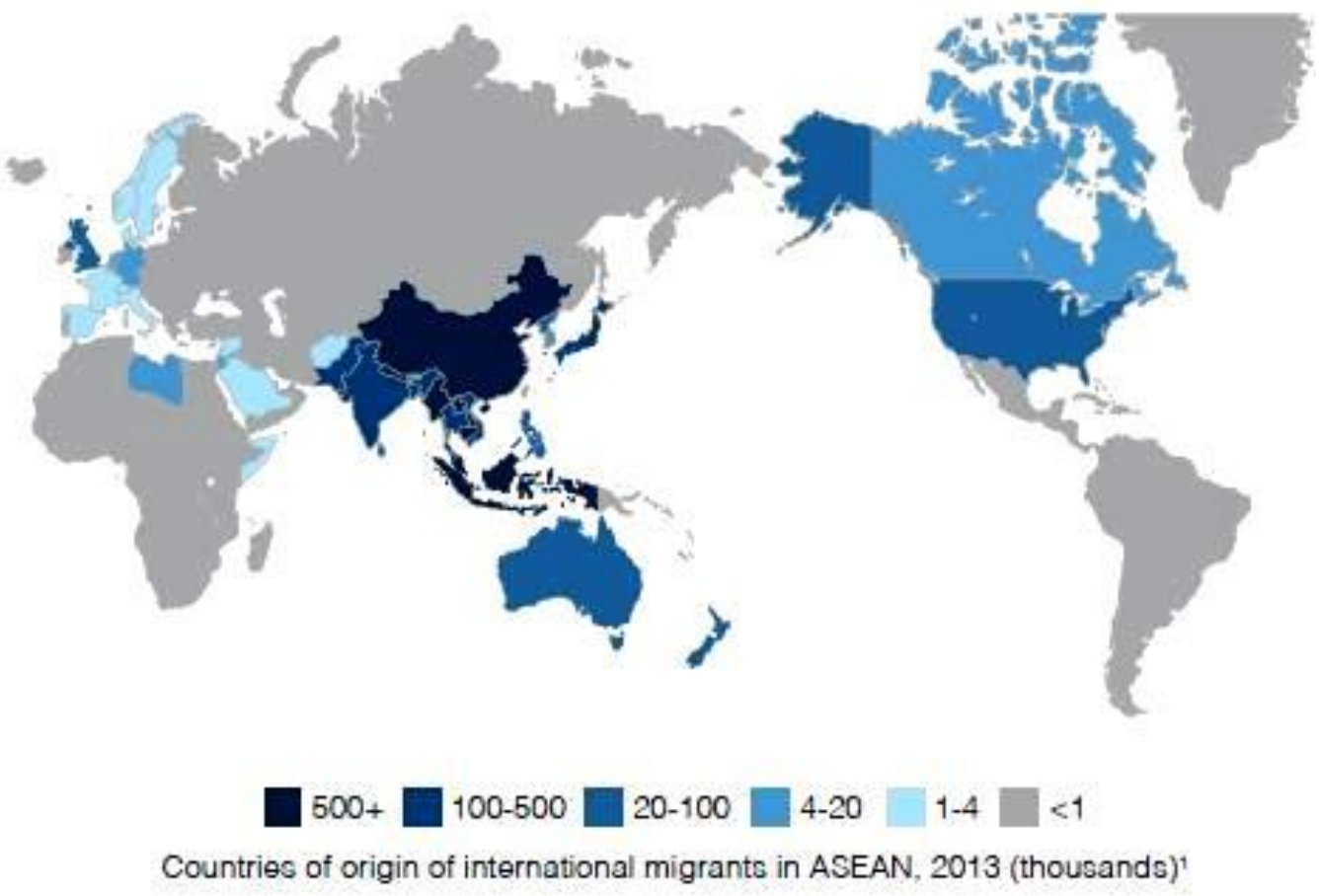

Source : OIT, pays d'origine et de destination des migrants dans l'ASEAN (Countries of Origin and Destination for Migrants in ASEAN). La base de données des statistiques internationales sur les migrations de main-d'œuvre (ILMS) pour l'ASEAN

Au début, la politique de travail des migrants de l'ASEAN était régie par son orientation économique néolibérale pour compléter sa stratégie d'ouverture commerciale et d'investissement, par exemple en encourageant le commerce des services. Selon cette «idéologie de marché » qui faisait partie intégrante de l'AEC, la libre circulation de la maind'œuvre garantissait au mieux l'utilisation efficace et productive du capital humain. De plus, il a favorisé le transfert de connaissances au sein de la région, par ex. en attirant, en retenant et en faisant circuler la main-d'œuvre qualifiée de l'ASEAN en utilisant des réglementations spécifiques, par ex. pour faciliter la circulation des étudiants, des professionnels qualifiés et des touristes. L'accord-cadre de l'ASEAN sur les services (AFAS) avait déjà été signé en 
1997, et l'Accord complémentaire de l'ASEAN sur la circulation des personnes en 2012 pour libéraliser davantage le commerce des services dans la région. Cependant, les accords n'ont jamais été mis en œuvre. Les flux intrarégionaux de l'ASEAN étaient dus en grande partie à des accords bilatéraux entre les pays d'origine et d'accueil et aux lois sur le travail et l'immigration des pays d'accueil, plutôt qu'à des initiatives à l'échelle de l'ASEAN (Kikkawa \& Suan, 2019).

Graphique 20 : Vers où migrent les ressortissants de l'ASEAN ?

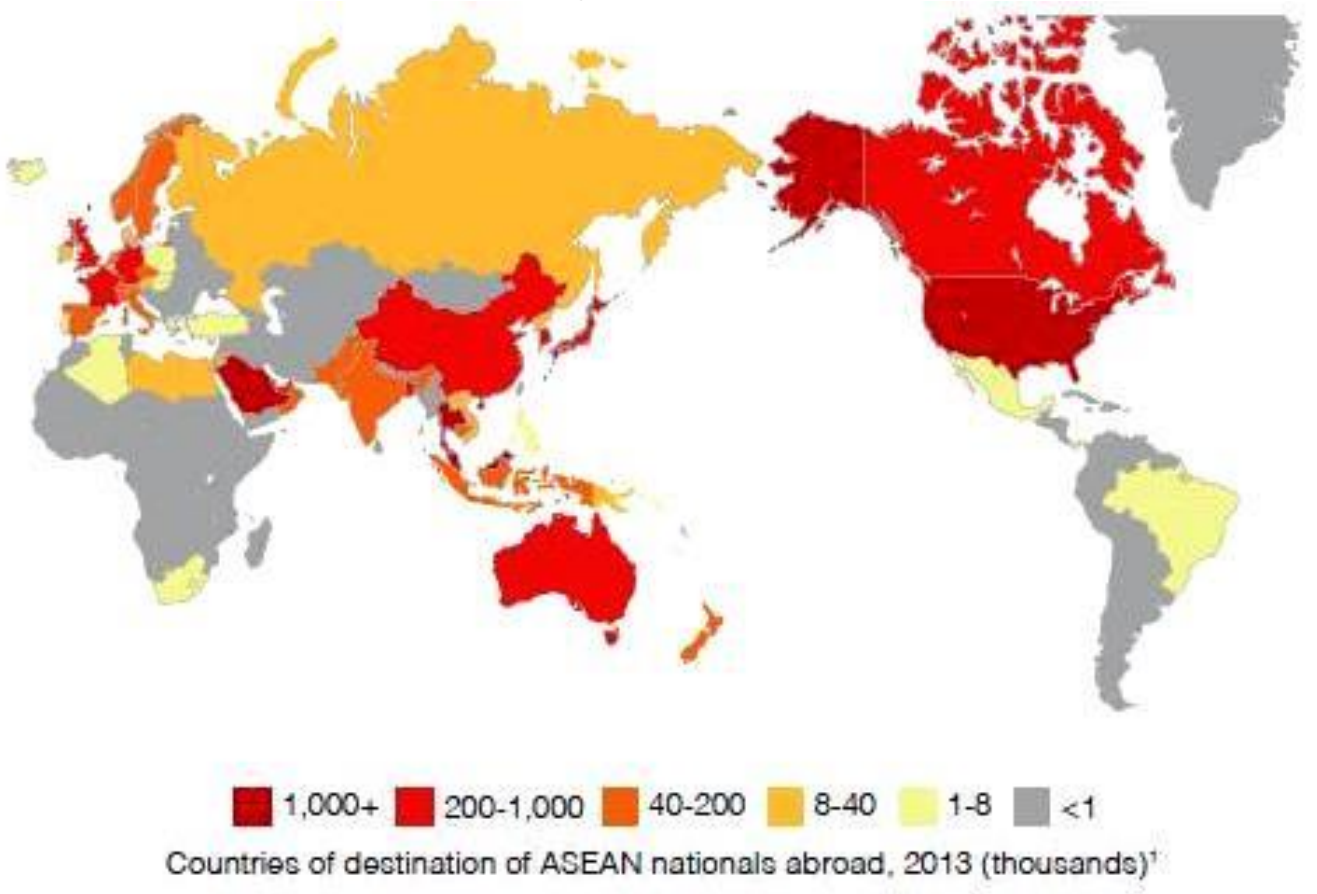

Source: ILO: $\underline{\text { Countries of Origin and Destination for Migrants in ASEAN }}$ ILMS Database for ASEAN

Graphique 21 : Femmes et hommes migrants internationaux dans l'ASEAN, 1960-2015

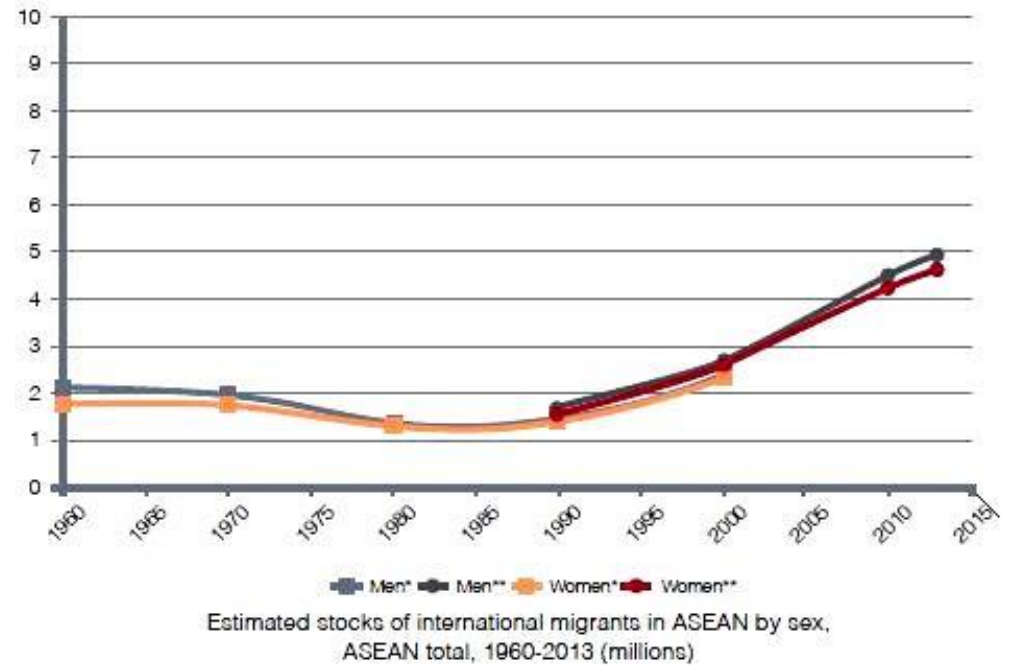

Source: ILO: Countries of Origin and Destination for Migrants in ASEAN ILMS Database for ASEAN 
L'étendue et l'échelle de la gouvernance étaient très différentes en ce qui concerne la maind'œuvre hautement qualifiée et peu qualifiée. Les ressources et le pouvoir de négociation inégaux des pays d'origine et d'accueil de l'ASEAN conduisent à des mesures de gouvernance inégales correspondantes. De plus, les progrès et la profondeur de la gouvernance étaient très inégaux, allant des réponses de haut en bas aux réponses envers (Rother, 2019). Les migrations ont été principalement motivées par des salaires plus élevés et des réseaux sociaux de migrants dans les économies d'accueil ainsi par des catastrophes naturelles dans les pays d'origine. En 2010, la Malaisie, l'Indonésie et la Thaïlande tentaient désespérément d'arrêter l'afflux de migrants en provenance du Myanmar et du Bangladesh. Ainsi, la marine indonésienne a refoulé les bateaux de migrants. Cependant, il serait injuste de blâmer uniquement le gouvernement de l'Indonésie, de la Malaisie et de la Thaïlande. Le Myanmar et le Bangladesh, ainsi que l'ASEAN comme organisation, ont également été responsables en raison de leur silence sur les violations des droits de l'homme, par ex. la discrimination systématique du régime du Myanmar à l'encontre des Rohingyas. D'autre part, les médias thaïlandais considéraient la traite des êtres humains comme un facteur clé pour que certaines superpuissances fassent pression sur le gouvernement de Bangkok par le biais de mesures commerciales (BBC-news, 2015).

Graphique 22 : Migration au sein des pays membres de l'ASEAN, 1995 et $2015^{6}$

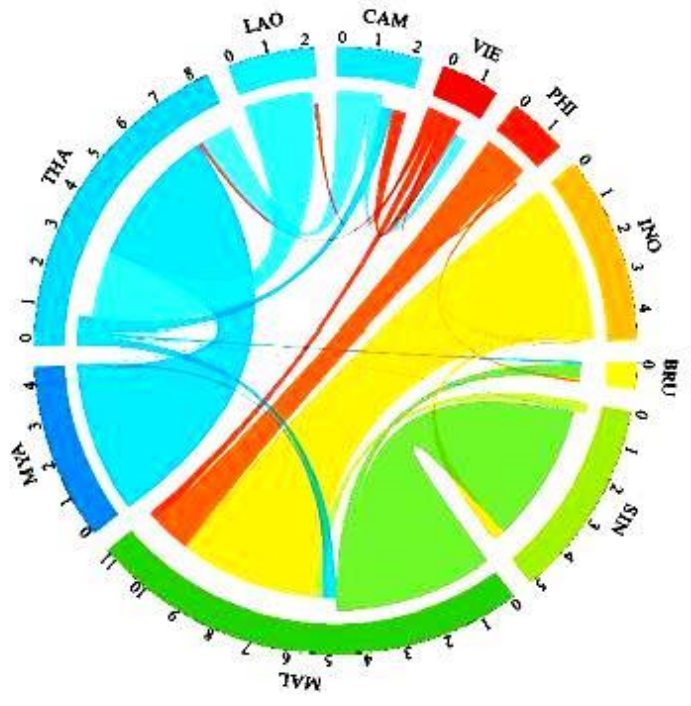

1995: Total migrant stock $=2.1$ million

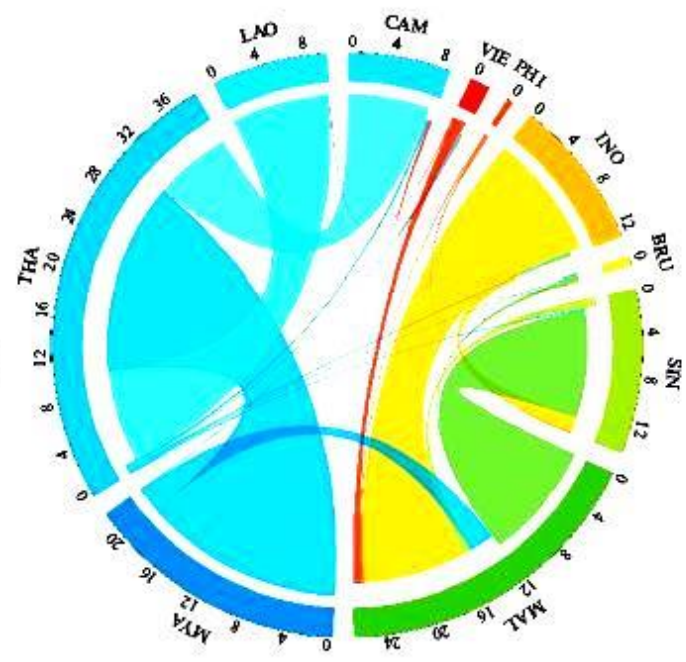

2015: Total migrant stock $=6.9$ million

Not $:$ BRU $=$ Brunei Darussalam; CAM $=$ Cambodia; $I N O=$ Indonesia; LAO $=$ Lao People's Democratic Republic; MAL $=$ Malaysia; MYA $=$ Myanmar; PHI = Philippines; SIN = Singapore; THA = Thailand $;$ VIE $=$ Viet Nam

Source: Authors' rendering based on Abel et.al. (2014), using the United Nations Department of Econo mic and Social Affairs (2015) database, Trends in International Migrant S tock: Migrants by Destination and Origin (United Nations data base, POP' DB'MIG/S tock'Rev.2015). Source: Kikkawa, Aiko \& Eric B. Suan 2019:7

Une grande partie de la migration était légale, mais une partie croissante de celle-ci a eu lieu en dehors du contrôle des gouvernements nationaux (Spaan \& Naerssen, 2018). Un nombre croissant de migrants vivaient dans un état de «transit» indéfini. Ils ont été profondément touchés par la situation incertaine et précaire. Néanmoins, beaucoup d'entre eux ont essayé de surmonter ces conditions et de poursuivre leurs conceptions de la vie, telles que se marier,

\footnotetext{
${ }^{6}$ Les cercles montrent les schémas de migration intra-ASEAN en 1995 et 2015. Ils sont basés sur les données de stock de migration bilatérale des Nations Unies. Chaque bande ou ligne représente le volume proportionnel et la direction de la migration (en stock) de la source (cercle extérieur) à l'hôte (cercle intérieur) (Kikkawa \&Suan, 2019:7).
} 
avoir des enfants, faire partie de la communauté locale et travailler pour un avenir meilleur. La conceptualisation du «transit » comme une vie dans les limbes ne rendait pas exactement compte de ces expériences permanentes qui changent la vie (Sampson \&Gifford, 2016).

Les sites de transit de migrants en Indonésie pour les migrants visant à se rendre en Australie ont illustré que les passages frontaliers étaient exploités non seulement dans des espaces illicites, mais aussi lorsqu'ils étaient sous le contrôle du gouvernement ou sous la gestion d'organisations internationales. Les économies de transit ont encouragé les réseaux informels s'attaquant aux migrants de transit à étendre leur exploitation (Phillips \& Missbach, 2017). Trois éléments structurels étaient susceptibles de conduire à davantage de migrations intraASEAN dans un avenir prévisible : la transition démographique, les grands écarts de revenus entre les économies de l'ASEAN et la porosité des frontières (Tuccio, 2017).

Le nombre de migrants se déplaçant sur le territoire de l'ASEAN a considérablement augmenté au cours des dernières décennies. La migration intra-ASEAN a triplé, passant de 2,1 millions en 1995 à 6,9 millions en 2015. Sa part du total des migrants dans l'ASEAN est passée de 57,6 \% en 1995 à 70,0 \% en 2015 (Kikkawa \& Suan, 2019). Cependant, le nombre de personnes cherchant refuge dans l'ASEAN à partir de sources extérieures a diminué de près de moitié, passant de 437,530 en 1980 à 284,949 en 2015, et la variété des nationalités est plus diversifiée que jamais.

Graphique 23 : Mobilité de la main-d'œuvre qualifiée de l'ASEAN et migration hors de la région (2015)

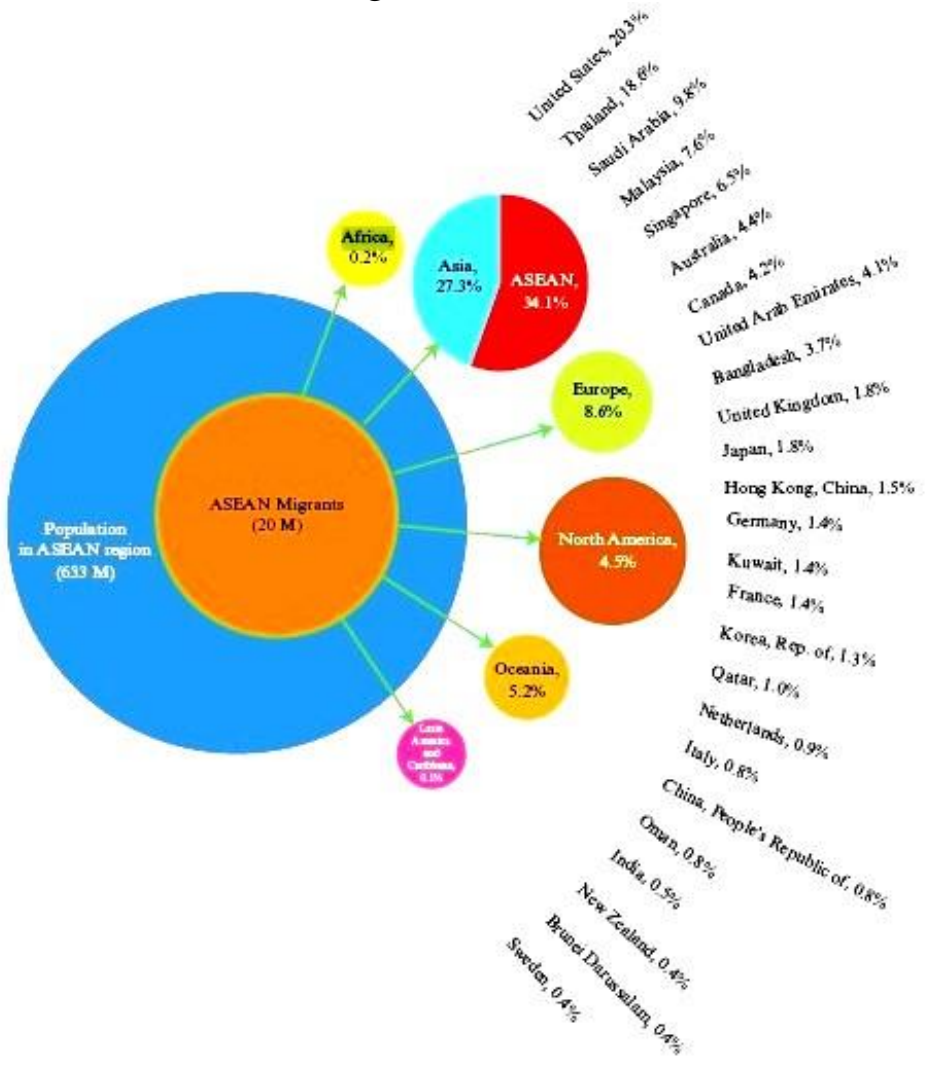

Note: $\quad \mathrm{M}=$ million people.

Sorce: Authors' calculations using the United Nations Department of Economic and Social Affairs database (2015), Trends in In ternational Migrant Stock: Migrants by Destination and Origin (United Nations database, POP/DB/MIG/Stock/Rev.2015).

Source: Kikkawa, Aiko \& Eric B. Suan (2019) 
En général, près de $80 \%$ des migrations Sud-Sud ont eu lieu entre des pays aux frontières contiguës. L'une des principales forces motrices était la recherche d'un meilleur revenu qui pourrait être partagé avec la famille qui a quitté le foyer. Les estimations des envois de fonds Sud-Sud allaient de $9 \%$ à $30 \%$ des envois de fonds des pays d'origine en 2005 . Il convient de mentionner que l'impact de la migration Sud-Sud sur le revenu des migrants était généralement plus faible que pour la migration Sud-Nord, et le coût des envois de fonds SudSud était encore plus élevé que celui des envois de fonds Nord-Sud. Néanmoins, même de légères augmentations de revenu pourraient avoir des effets substantiels sur le bien-être des pauvres. Enfin, les coûts des transferts Sud-Sud sont encore plus élevés que ceux des transferts Nord-Sud (Ratha \& Shaw, 2006).

La Malaisie, par exemple, est devenue un pays d'asile pour les migrants forcés originaires majoritairement d'Afrique, par ex. de l'Angola, du Burundi, de la République Centrafricaine, du Cameroun, de la République démocratique du Congo, de l'Algérie, de la Guinée, de l'Éthiopie, du Kenya, du Rwanda et du Sénégal, mais aussi du Bhoutan, de l'Iran, de l'Irak et du Koweït. Des tendances similaires de migration forcée Africaines sont apparues en Thaillande et en Indonésie, qui ont connu une plus grande diversification des nationalités par rapport à la période d'avant les années 1990. L'ASEAN serait bien avisée d'établir une coopération stratégique, axée sur les problèmes de migration forcée avec l'Union africaine, la Commission africaine des droits de l'homme et des peuples (CADHP) et l'Organisation de la coopération islamique (OCI) (Wahab, 2017). Les pays de l'ASEAN ayant enregistré des flux migratoires nets étaient le Brunei, la Malaisie, Singapour et la Thaïlande. Parmi les économies avec des sorties nettes figuraient le Cambodge, l'Indonésie, le Laos, le Myanmar, les Philippines et le Viêtnam (Kikkawa \& Suan, 2019). Les migrants d'Afrique subsaharienne, par exemple en Thaïlande, étaient en outre handicapés par le manque de maîtrise de la langue thaï et l'accès limité aux soins de santé. De plus, ce dernier était dû au manque de compétences interculturelles et à la méfiance à l'égard du personnel de santé thaïlandais, et à l'absence de services de traduction dans la plupart des établissements de santé thaïlandais (Gonah, 2016).

\section{Conclusion}

L'analyse de la performance économique des États membre de l'ASEAN en Afrique est compliquée en raison de la forte diversité politique, économique, sociale et culturelle de l'ASEAN et de l'Afrique. Des réponses unidimensionnelles seraient trompeuses. Les études de cas par pays ont montré qu'elles offraient une alternative convaincante. Des études approfondies sur les performances du Nouveau partenariat stratégique Asie-Afrique (NAASP), en prenant l'exemple de deux pays de l'ASEAN (Indonésie et Thaïlande) ainsi que de trois pays africains (Afrique du Sud, Nigéria, Égypte), ont révélé des informations pertinants.

Bien que les positions controversées sur les violations des droits humains du régime militaire autocratique au Myanmar et les différentes exigences de suzeraineté concernant la mer de Chine méridionale aient entravé les relations politiques internes de l'ASEAN, cela n'a eu que peu ou pas d'effet sur les relations africaines de l'ASEAN. Alors qu'à l'époque de la guerre froide, la solidarité entre les «nations du tiers-monde » et les colonies a soudé des liens quasi «naturels » entre l'Asie et l'Afrique, comme cristallisé dans la déclaration de Bandung, ce sentiment d'un destin commun a diminué par la suite. La création du Nouveau partenariat stratégique Asie-Afrique (NAASP) en 2005 visait à revitaliser l'esprit de Bandung du 
mouvement des non-alignés, en mettant davantage l'accent sur les avantages mutuels de la coopération économique. Le commerce ASEAN-Afrique est passé de seulement 2,8 milliards US\$ en 1990 à 42,5 milliards US\$ en 2012, augmentant ainsi en moyenne de $14 \%$ par an.

Cependant, étant donné le déplacement des réseaux mondiaux de puissance politique et militaire de la région Atlantique vers la région Pacifique, et en raison du développement rapide des pays membres de l'ASEAN face à la croissance atone de l'Afrique, ainsi qu'à la crise politique et sécuritaire permanente en ASS, l'intérêt des investisseurs et commerçants de l'ASEAN vis-à-vis de leurs homologues africains se sont affaiblis.

En outre, la pandémie de Coronavirus et les effets associés, comme les perturbations ultérieures des chaînes d'approvisionnement, ont gravement affecté la croissance globale du PIB, l'emploi, le commerce extérieur et le bien-être, à la fois dans l'ASEAN et en Afrique (Kohnert, 2021; 2021a). En novembre 2020, le nombre de cas de COVID-19 reconnus dans les 10 États de l'ANASE avait dépassé le million. L'Indonésie et les Philippines ont pris la tête avec plus de 440,500 et 398,400 cas. Les décès confirmés liés à COVID-19 à cette époque étaient de plus de 24,100, la plupart en Indonésie (Vu, 2020). Plus tard, le Viêtnam a été le plus durement touché par COVID-19, en particulier depuis le début de la quatrième vague en cours des fin avril 2021, de nombreuses activités socio-économiques s'arrêtant pendant des mois, ou du moins ayant été perturbées de temps en temps. La Chine a fourni aux États membres de l'ASEAN, dont le Cambodge, le Laos et le Myanmar, plus de 300 millions de doses de vaccins contre le COVID-19 ainsi qu'un grand nombre de fournitures médicales d'urgence (Xinhua, 2021a). À l'été 2021, les pays de l'ANASE ont commencé à rouvrir leurs économies et leurs frontières suite au recul des activités économiques et sociales pendant l'épidémie de COVID-19. Cependant, l'impact réel sur les relations ASEAN-Afrique n'a pas encore été analysé en raison du manque de données fiables. Au total, ces évolutions ont rendu les perspectives de coopération ASEAN-Afrique, dont le NAASP, encore moins prometteuses dans un avenir prévisible. 


\section{References}

AFR-IX (2021): Comparison of Foreign Countries Direct Investment in Africa. AFR-IXtelecom, 28 September 2021

Albert, Melissa (2021): Bandung Conference, Asia-Africa [1955], Encyclopæedia Britannica

Aljazeera (2021): ASEAN summit begins without Myanmar after top general barred. 26 Oct 2021

Alatas, Ali (2005): Towards a New Strategic Partnership between Asia and Africa. (RSIS Commentaries, No. 018). RSIS Commentaries. Singapore: Nanyang Technological University. https://hdl.handle.net/10356/82272

Austine, Ifeanyi Okere (2019): African Economic Growth and Development: Lessons from the Association of Southeast Asian Nations (ASEAN). International Journal of Innovative Research and Development, March 2019 Vol 8 Issue 3 ISSN 2278 - 0211 (Online) / Nigerian Journal Online

BBC-news (2015): Asia boat migrants: Asean media troubled over treatment. No author given, $B B C$ news, 18 May 2015

Coulibaly, Souleymane (2020): Two lessons from ASEAN for regional integration in Africa. Brookings.edu, July 29, 2020

Dhawan, Vivek (2017): What next for Thailand-Africa relations? Bangkok Post, 16 February 2015

Diadié Diaw, Thi Anh-Dao Tran (2020): Les relations commerciales entre l'Asie du Sud-Est et l'Afrique subsaharienne: les promesses d'une coopération Sud-Sud. In: Cabasset, Christine et al (2020): L'Asie du Sud-Est 2020. Paris: 456 p.

Dlamini, Kenny (2019): Building Asia-Africa Cooperation: Analysing the Relevance of the New Asia-Africa Strategic Partnership (NAASP). Pretoria: UNISA Institute for Global Dialogue (IGD), Africa-Portal, 4 Nov 2019

Dlamini, Kenny (2021): South Africa and ASEAN countries: the need for strategic partnerships. Pretoria: UNISA, Insitute for Global Dialogue

Dorigné-Thomson, Christophe (2021): Indonesia's African Foreign Policy Shift under President Jokowi. Journal of Social and Political Sciences, Vol.4 No.3 (2021), Available at SSRN: https://ssrn.com/abstract=3900546

Egypt Today staff (2020): Trade exchange bet. Egypt, Viêtnam records \$491M in 2019. Egypt Today, 20 Dec 2020

Fähnders, Till (2021): Zwischen China und Amerika - Die ASEAN-Staaten füchten eine wachsende Konfrontation in Asien. Frankfurter Allgemeine Zeitung (faz), 28 October 2021

Fisher, Hilton (2020): SA agreement with South East Asian bloc opens up a host of opportunities, Pretoria: Department of International Relations and Cooperation, Daily Maverick, 30 September 2020

FRANCE-24 (2021): ASEAN summit opens without snubbed Myanmar junta chief. Paris: 24 October 2021

Gaens, Bart \& Bernardo Venturi \& Anna Ayuso (2020): Differentiation in ASEAN, ECOWAS, and MERCOSUR: A Comparative Analysis. EUIDEA Policy Papers, August 2020 
GIS-Asie (2016): ASEAN-Africa: Towards a Renewed Partnership. French Academic Network on Asian Studies, Chulalongkorn University, ASEAN Studies Center, International Workshop, 29th January 2016

Gonah, L. et al (2016): Barriers to Healthcare Access and Coping Mechanisms among Sub-Saharan African Migrants living in Bangkok, Thailand: A Qualitative Study. Medical Journal of Zambia, Vol. 43 (4): $238-246$

Kohnert, Dirk (2021): The socio-economic impact of Brexit on India, Pakistan, and Sri Lanka in times of Corona. MPRA-WP, Nr. 108822

Kohnert, Dirk (2021): The socio-economic impact of Brexit on CANZUK and the Anglosphere in times of Corona: The case of UK, Canada, Australia, and New Zealand. SSRN-WPS, Nr. 3854401

Loong, Lee Hsien (2021a): Integration in ein neues Asien. Wie Chinas Aufstieg die internationale Architektur verändert. Internationale Politik, No. 6, 2021

Medina, Ayman Falak (2020): More Countries Join ASEAN's Treaty of Amity and Cooperation. ASEAN Briefing, November 20, 2020

Ministry of Foreign Affairs, Jakarta (2019): Indonesia - Egypt Agree to Raise Economic and Consular Partnership. 28 June 2019

Okeh, Victor (2018): Nigeria, ASEAN Bilateral Trade Relations Reaches \$7.7 billion. BizWatch Nigeria, August 7, 2018

Phillips, Melissa \& Antje Missbach (2017): Economies of transit: exploiting migrants and refugees in Indonesia and Libya. Migration and Border Studies, March 24, 2017, pp 139-157

Ratha, Dilip \& William Shaw (2006): South-South Migration and Remittances. Washington D.C.: World Bank, Development Prospects Group, December 1, 2006

Rensburg, Susara J. Jansen van \& Wilma Viviers \& Martin Cameron \& Ali Parry (2019): Identifying export opportunities between IORA member states using the TRADE-DSM $®$ methodology: a case study involving South Africa and Thailand. Journal of the Indian Ocean Region, 15:1, 78-96, DOI: 10.1080/19480881.2018.1521777

Rother, Stefan (2019): The uneven migration governance of ASEAN. In: Geddes, A. et al (eds.): The Dynamics of Regional Migration Governance, pp. 186-204

Sampson, Robyn C. \& Sandra M. Gifford (2016): The myth of transit: the making of a life by asylum seekers and refugees in Indonesia. Journal of Ethnic and Migration Studies, Volume 42, 2016 Issue 7 Pages 1135-1152

Sermcheep, Sineenat (2017): The Rise of Outward Foreign Direct Investment from ASEAN. In: Lee, Cassey \& Sineenat Sermcheep (eds.): Outward Foreign Direct Investment in ASEAN. Singapore: ISEAS Publishing, 2017, pp. 5-29

Spaan, Ernst \& Ton van Naerssen (2018): Migration decision-making and migration industry in the Indonesia-Malaysia corridor. Journal of Ethnic and Migration Studies, 44:4, 680-695

SAWIT (2018): Egypt Could be the entrance for Indonesian palm oil to African market. Jakarta: BPDP Sawit - Beranda, Jun 27, 2018

Thai's Ministry of Foreign Affairs (2021): Permanent Secretary Strengthened Thailand-Egypt Cordial Relationship with Ambassador of Egypt, Focusing on Cooperation on Education, Trade \& Investment as well as Fishery. Ministry of Foreign Affairs, Kingdom of Thailand. , Bangkok, 18 Mar 2021

Tang, Heiwai \& Douglas Zhihua Zeng \&Albert Zeufac (2020): Assessing Asia - Sub-Saharan Africa global value chain linkages. Kiel: IfW, Kiel-WP No. 2159, June 2020 
Tanuwidjaja, Enrico \& Haris Handy (2021): Assessing Indonesia-Africa Trade Opportunities: Mapping of Competitiveness and Specialization. International Economic Association (IEA), World Congress, 2-6 July 2021

Tarrósy, István (2016): Indonesia in Africa: Revitalizing Relations. Univ. of Leiden, African Studies Centre, ASC Infosheet No. 29

Tuccio, Michele (2017): Determinants of Intra-ASEAN Migration. Asian Development Review (2017) 34 (1): 144-166

Vaidyanathan, Veda (2016): Exploring trade and investment patterns of ASEAN in Africa: Are they limited by the bigger Asian powers? New Delhi: Institute of Chines Studies, Occasional Papers, No.13, November 2016

VNA (2020): Viêtnam - emerging tiger in Asia: Egyptian media. Viêtnam plus VN

Vu, Phuong (2020): ASEAN Summit to discuss pandemic control, aftermath. VN-Express international, November 10, 2020

Wahab, Andika Ab. (2017): The Future of Forced Migrants in ASEAN. Bonn: Heinrich Boell Foundation, 2 August 2017

Williams, Christopher \& Mihaela Papa (2020): Rethinking "Alliances": The Case of South Africa as a Rising Power, African Security, 13:4, 325-352, DOI: 10.1080/19392206.2020.1871796

Yazid, Sylvia \& Iyan Septiyana (2019): The prospects of ASEAN migration governance. Journal of Indonesian Social Science and Humanities, vol 9, no 2, pp. 2656-7512

Xinhua (2021): RCEP to boost ASEAN's economic recovery from COVID-19, says Singapore scholar. Beijing: Xinhua, 25 October 2021

Xinhua (2021a): China, ASEAN join hands in fighting COVID-19, boosting economic recovery. Beijing: Source: Xinhua, 26 October 2021 


\begin{abstract}
ASEAN - African relations. Towards a new partnership ? ----- The ASEAN summit of October 2021 showed the increased geopolitical importance of the Indo-Pacific realm. Today ASEAN is the most successful regional organization in Asia and the second largest worldwide behind the EU. The establishment of the New Asian-African Strategic Partnership (NAASP) more than 15 years before (2005) aimed to revive the Bandung spirit of the non-aligned movement of 1955. This time with a stronger focus on economic ties. In 2013 these countries counted around 620 million inhabitants or $8.8 \%$ of the world population. They wanted to fight colonialism and neocolonialism by promoting Afro-Asiatic economic and cultural cooperation. Almost all member countries gained sovereignty and political independence by the 1960s and 1970s, except for Palestine. However, the aftermath of the Bandung conference also promoted negative developments, including the polarization of Asian countries, the strengthening of political authoritarianism, and regional interventions. In addition, most countries continued to grapple with economic and political challenges, including poverty, debt burdens, backwardness, ignorance, disease, and environmental degradation. Their access to the markets of the industrialized countries also remained limited. At the global level, the NAASP received little attention so far. Despite the longstanding rhetoric of Asia-Africa solidarity, Asia and Africa still lack formal institutional and trade links. Although interregional trade increased, Africa remained a small part of ASEAN with only around $2 \%$ of its total market. The most important trading countries of ASEAN with Africa were Thailand, Indonesia, and Singapore, while South Africa, Nigeria, and Egypt were the largest African import markets.
\end{abstract}

Zusammenfassung:Die Beziehungen zwischen ASEAN und Afrika: Aufbruch zu einer erneuerten Partnerschaft ? ---- Der ASEAN-Gipfel vom Oktober 2021 zeigte die zunehmende geopolitische Bedeutung des Indopazifischen Raums. Heute ist ASEAN die erfolgreichste Regionalorganisation in Asien und die zweitgrößte weltweit hinter der EU. Die Gründung der New Asian-African Strategic Partnership (NAASP) vor mehr als 15 Jahren (2005) hatte das Ziel, den Bandung-Geist der blockfreien Bewegung von 1955 wiederzubeleben. Diesmal mit einem stärkeren Fokus auf wirtschaftliche Verbindungen. Im Jahr 2013 zählten diese Länder rund 620 Millionen Einwohner oder 8,8\% der Weltbevölkerung. Sie wollten Kolonialismus und Neokolonialismus bekämpfen, indem sie die afro-asiatische wirtschaftliche und kulturelle Zusammenarbeit förderten. Fast alle Mitgliedsländer, mit Ausnahme Palästinas, erlangten in den 1960er und 1970er Jahren Souveränität und politische Unabhängigkeit. Die Nachwirkungen der Bandung-Konferenz förderten jedoch auch negative Entwicklungen, darunter die Polarisierung asiatischer Länder, die Stärkung des politischen Autoritarismus und regionale Interventionen. Darüber hinaus kämpften die meisten Länder weiterhin mit wirtschaftlichen und politischen Herausforderungen, darunter Armut, Schuldenlast, Rückständigkeit, Ignoranz, Krankheiten und Umweltzerstörung. Auch ihr Zugang zu den Märkten der Industrieländer blieb begrenzt. Auf globaler Ebene wurde der NAASP bisher wenig Aufmerksamkeit geschenkt. Trotz der langjährigen Rhetorik der Solidarität zwischen Asien und Afrika fehlt es Asien und Afrika immer noch an formellen institutionellen und handelspolitischen Verbindungen. Obwohl der interregionale Handel zunahm, blieb Afrika ein kleiner Teil der ASEAN mit nur rund $2 \%$ seines Gesamtmarktes. Die wichtigsten Handelsländer der ASEAN mit Afrika waren Thailand, Indonesien und Singapur, während Südafrika, Nigeria und Ägypten die größten afrikanischen Importmärkte waren. 